DESY-08-056

29 May 2008

\title{
Beauty photoproduction using decays into electrons at HERA
}

\author{
ZEUS Collaboration
}

\begin{abstract}
Photoproduction of beauty quarks in events with two jets and an electron associated with one of the jets has been studied with the ZEUS detector at HERA using an integrated luminosity of $120 \mathrm{pb}^{-1}$. The fractions of events containing $b$ quarks, and also of events containing $c$ quarks, were extracted from a likelihood fit using variables sensitive to electron identification as well as to semileptonic decays. Total and differential cross sections for beauty and charm production were measured and compared with next-to-leading-order QCD calculations and Monte Carlo models.
\end{abstract}




\section{The ZEUS Collaboration}

S. Chekanov, M. Derrick, S. Magill, B. Musgrave, D. Nicholass ${ }^{1}$, J. Repond, R. Yoshida Argonne National Laboratory, Argonne, Illinois 60439-4815, USA ${ }^{n}$

M.C.K. Mattingly

Andrews University, Berrien Springs, Michigan 49104-0380, USA

P. Antonioli, G. Bari, L. Bellagamba, D. Boscherini, A. Bruni, G. Bruni, F. Cindolo, M. Corradi, G. Iacobucci, A. Margotti, R. Nania, A. Polini INFN Bologna, Bologna, Italy ${ }^{e}$

S. Antonelli, M. Basile, M. Bindi, L. Cifarelli, A. Contin, S. De Pasquale ${ }^{2}$, G. Sartorelli, A. Zichichi University and INFN Bologna, Bologna, Italy ${ }^{e}$

D. Bartsch, I. Brock, H. Hartmann, E. Hilger, H.-P. Jakob, M. Jüngst, A.E. Nuncio-Quiroz, E. Paul, U. Samson, V. Schönberg, R. Shehzadi, M. Wlasenko Physikalisches Institut der Universität Bonn, Bonn, Germany ${ }^{b}$

N.H. Brook, G.P. Heath, J.D. Morris

H.H. Wills Physics Laboratory, University of Bristol, Bristol, United Kingdom ${ }^{m}$

M. Capua, S. Fazio, A. Mastroberardino, M. Schioppa, G. Susinno, E. Tassi

Calabria University, Physics Department and INFN, Cosenza, Italy ${ }^{e}$

J.Y. Kim

Chonnam National University, Kwangju, South Korea

Z.A. Ibrahim, B. Kamaluddin, W.A.T. Wan Abdullah

Jabatan Fizik, Universiti Malaya, 50603 Kuala Lumpur, Malaysia ${ }^{r}$

Y. Ning, Z. Ren, F. Sciulli

Nevis Laboratories, Columbia University, Irvington on Hudson, New York 10027o

J. Chwastowski, A. Eskreys, J. Figiel, A. Galas, M. Gil, K. Olkiewicz, P. Stopa, L. Zawiejski The Henryk Niewodniczanski Institute of Nuclear Physics, Polish Academy of Sciences, Cracow, Poland ${ }^{i}$

L. Adamczyk, T. Bołd, I. Grabowska-Bołd, D. Kisielewska, J. Łukasik, M. Przybycień, L. Suszycki

Faculty of Physics and Applied Computer Science, AGH-University of Science and Technology, Cracow, Poland ${ }^{p}$

A. Kotański ${ }^{3}$, W. Słomiński ${ }^{4}$

Department of Physics, Jagellonian University, Cracow, Poland 
U. Behrens, C. Blohm, A. Bonato, K. Borras, R. Ciesielski, N. Coppola, S. Fang, J. Fourletova ${ }^{5}$, A. Geiser, P. Göttlicher ${ }^{6}$, J. Grebenyuk, I. Gregor, T. Haas, W. Hain, A. Hüttmann, F. Januschek, B. Kahle, I.I. Katkov, U. Klein ${ }^{7}$, U. Kötz, H. Kowalski, E. Lobodzinska, B. Löhr, R. Mankel, I.-A. Melzer-Pellmann, S. Miglioranzi, A. Montanari, T. Namsoo, D. Notz ${ }^{8}$, A. Parenti, L. Rinaldi ${ }^{9}$, P. Roloff, I. Rubinsky, R. Santamarta ${ }^{10}$, U. Schneekloth, A. Spiridonov ${ }^{11}$, D. Szuba ${ }^{12}$, J. Szuba ${ }^{13}$, T. Theedt, G. Wolf, K. Wrona, A.G. Yagües Molina, C. Youngman, W. Zeuner ${ }^{8}$ Deutsches Elektronen-Synchrotron DESY, Hamburg, Germany

V. Drugakov, W. Lohmann, S. Schlenstedt

Deutsches Elektronen-Synchrotron DESY, Zeuthen, Germany

G. Barbagli, E. Gallo

INFN Florence, Florence, Italy ${ }^{e}$

P. G. Pelfer

University and INFN Florence, Florence, Italy $^{e}$

A. Bamberger, D. Dobur, F. Karstens, N.N. Vlasov ${ }^{14}$

Fakultät für Physik der Universität Freiburg i.Br., Freiburg i.Br., Germany ${ }^{b}$

P.J. Bussey ${ }^{15}$, A.T. Doyle, W. Dunne, M. Forrest, M. Rosin, D.H. Saxon, I.O. Skillicorn Department of Physics and Astronomy, University of Glasgow, Glasgow, United Kingdom ${ }^{m}$

I. Gialas ${ }^{16}$, K. Papageorgiu

Department of Engineering in Management and Finance, Univ. of Aegean, Greece

U. Holm, R. Klanner, E. Lohrmann, P. Schleper, T. Schörner-Sadenius, J. Sztuk, H. Stadie, M. Turcato

Hamburg University, Institute of Exp. Physics, Hamburg, Germany ${ }^{b}$

C. Foudas, C. Fry, K.R. Long, A.D. Tapper

Imperial College London, High Energy Nuclear Physics Group, London, United Kingdom ${ }^{m}$

T. Matsumoto, K. Nagano, K. Tokushuku ${ }^{17}$, S. Yamada, Y. Yamazaki ${ }^{18}$

Institute of Particle and Nuclear Studies, KEK, Tsukuba, Japan ${ }^{f}$

A.N. Barakbaev, E.G. Boos, N.S. Pokrovskiy, B.O. Zhautykov

Institute of Physics and Technology of Ministry of Education and Science of Kazakhstan, Almaty, Kazakhstan

V. Aushev ${ }^{19}$, M. Borodin, I. Kadenko, A. Kozulia, V. Libov, M. Lisovyi, D. Lontkovskyi, I. Makarenko, Iu. Sorokin, A. Verbytskyi, O. Volynets

Institute for Nuclear Research, National Academy of Sciences, Kiev and Kiev National University, Kiev, Ukraine 
D. Son

Kyungpook National University, Center for High Energy Physics, Daegu, South Korea ${ }^{g}$

J. de Favereau, K. Piotrzkowski

Institut de Physique Nucléaire, Université Catholique de Louvain, Louvain-la-Neuve, Belgium ${ }^{q}$

F. Barreiro, C. Glasman, M. Jimenez, L. Labarga, J. del Peso, E. Ron, M. Soares,

J. Terrón, M. Zambrana

Departamento de Física Teórica, Universidad Autónoma de Madrid, Madrid, Spain ${ }^{l}$

F. Corriveau, C. Liu, J. Schwartz, R. Walsh, C. Zhou

Department of Physics, McGill University, Montréal, Québec, Canada H3A 2T8 ${ }^{a}$

T. Tsurugai

Meiji Gakuin University, Faculty of General Education, Yokohama, Japan ${ }^{f}$

A. Antonov, B.A. Dolgoshein, D. Gladkov, V. Sosnovtsev, A. Stifutkin, S. Suchkov

Moscow Engineering Physics Institute, Moscow, Russia ${ }^{j}$

R.K. Dementiev, P.F. Ermolov ${ }^{\dagger}$, L.K. Gladilin, Yu.A. Golubkov, L.A. Khein, I.A. Korzhavina, V.A. Kuzmin, B.B. Levchenko ${ }^{20}$, O.Yu. Lukina, A.S. Proskuryakov, L.M. Shcheglova, D.S. Zotkin

Moscow State University, Institute of Nuclear Physics, Moscow, Russia ${ }^{k}$

I. Abt, A. Caldwell, D. Kollar, B. Reisert, W.B. Schmidke

Max-Planck-Institut für Physik, München, Germany

G. Grigorescu, A. Keramidas, E. Koffeman, P. Kooijman, A. Pellegrino, H. Tiecke, M. Vázquez ${ }^{8}$, L. Wiggers

NIKHEF and University of Amsterdam, Amsterdam, Netherlands ${ }^{h}$

N. Brümmer, B. Bylsma, L.S. Durkin, A. Lee, T.Y. Ling

Physics Department, Ohio State University, Columbus, Ohio 43210 ${ }^{n}$

P.D. Allfrey, M.A. Bell, A.M. Cooper-Sarkar, R.C.E. Devenish, J. Ferrando, B. Foster, K. Korcsak-Gorzo, K. Oliver, S. Patel, V. Roberfroid ${ }^{21}$, A. Robertson, P.B. Straub, C. Uribe-Estrada, R. Walczak

Department of Physics, University of Oxford, Oxford United Kingdom ${ }^{m}$

A. Bertolin, F. Dal Corso, S. Dusini, A. Longhin, L. Stanco

INFN Padova, Padova, Italy ${ }^{e}$

P. Bellan, R. Brugnera, R. Carlin, A. Garfagnini, S. Limentani

Dipartimento di Fisica dell' Università and INFN, Padova, Italy ${ }^{e}$

B.Y. Oh, A. Raval, J. Ukleja ${ }^{22}$, J.J. Whitmore ${ }^{23}$

Department of Physics, Pennsylvania State University, University Park, Pennsylvania $16802^{\circ}$ 
Y. Iga

Polytechnic University, Sagamihara, Japan ${ }^{f}$

G. D'Agostini, G. Marini, A. Nigro

Dipartimento di Fisica, Università 'La Sapienza' and INFN, Rome, Italy ${ }^{e}$

J.E. Cole ${ }^{24}$, J.C. Hart

Rutherford Appleton Laboratory, Chilton, Didcot, Oxon, United Kingdom ${ }^{m}$

H. Abramowicz ${ }^{25}$, R. Ingbir, S. Kananov, A. Levy, A. Stern

Raymond and Beverly Sackler Faculty of Exact Sciences, School of Physics, Tel Aviv

University, Tel Aviv, Israel ${ }^{d}$

M. Kuze, J. Maeda

Department of Physics, Tokyo Institute of Technology, Tokyo, Japan ${ }^{f}$

R. Hori, S. Kagawa ${ }^{26}$, N. Okazaki, S. Shimizu, T. Tawara

Department of Physics, University of Tokyo, Tokyo, Japan ${ }^{f}$

R. Hamatsu, H. Kaji ${ }^{27}$, S. Kitamura ${ }^{28}$, O. Ota ${ }^{29}$, Y.D. Ri

Tokyo Metropolitan University, Department of Physics, Tokyo, Japan ${ }^{f}$

M. Costa, M.I. Ferrero, V. Monaco, R. Sacchi, A. Solano

Università di Torino and INFN, Torino, Italy ${ }^{e}$

M. Arneodo, M. Ruspa

Università del Piemonte Orientale, Novara, and INFN, Torino, Italy ${ }^{e}$

S. Fourletov ${ }^{5}$, J.F. Martin, T.P. Stewart

Department of Physics, University of Toronto, Toronto, Ontario, Canada M5S 1A7 a

S.K. Boutle ${ }^{16}$, J.M. Butterworth, C. Gwenlan ${ }^{30}$, T.W. Jones, J.H. Loizides, M. Wing ${ }^{31}$ Physics and Astronomy Department, University College London, London, United Kingdom ${ }^{m}$

B. Brzozowska, J. Ciborowski ${ }^{32}$, G. Grzelak, P. Kulinski, P. Łużniak ${ }^{33}$, J. Malka ${ }^{33}$, R.J. Nowak, J.M. Pawlak, T. Tymieniecka, A. Ukleja, A.F. Żarnecki

Warsaw University, Institute of Experimental Physics, Warsaw, Poland

M. Adamus, P. Plucinski ${ }^{34}$

Institute for Nuclear Studies, Warsaw, Poland

Y. Eisenberg, D. Hochman, U. Karshon

Department of Particle Physics, Weizmann Institute, Rehovot, Israel ${ }^{c}$

E. Brownson, T. Danielson, A. Everett, D. Kçira, D.D. Reeder, P. Ryan, A.A. Savin, W.H. Smith, H. Wolfe

Department of Physics, University of Wisconsin, Madison, Wisconsin 53706, USA ${ }^{n}$

S. Bhadra, C.D. Catterall, Y. Cui, G. Hartner, S. Menary, U. Noor, J. Standage, J. Whyte

Department of Physics, York University, Ontario, Canada M3J 1P3 ${ }^{a}$ 
1 also affiliated with University College London, UK

2 now at University of Salerno, Italy

${ }^{3}$ supported by the research grant no. 1 P03B 04529 (2005-2008)

4 This work was supported in part by the Marie Curie Actions Transfer of Knowledge project COCOS (contract MTKD-CT-2004-517186)

${ }^{5}$ now at University of Bonn, Germany

${ }^{6}$ now at DESY group FEB, Hamburg, Germany

7 now at University of Liverpool, UK

8 now at CERN, Geneva, Switzerland

${ }^{9}$ now at Bologna University, Bologna, Italy

10 now at BayesForecast, Madrid, Spain

11 also at Institut of Theoretical and Experimental Physics, Moscow, Russia

12 also at INP, Cracow, Poland

13 also at FPACS, AGH-UST, Cracow, Poland

14 partly supported by Moscow State University, Russia

${ }^{15}$ Royal Society of Edinburgh, Scottish Executive Support Research Fellow

16 also affiliated with DESY, Germany

17 also at University of Tokyo, Japan

18 now at Kobe University, Japan

19 supported by DESY, Germany

20 partly supported by Russian Foundation for Basic Research grant no. 05-02-39028NSFC-a

${ }^{21}$ EU Marie Curie Fellow

22 partially supported by Warsaw University, Poland

23 This material was based on work supported by the National Science Foundation, while working at the Foundation.

${ }^{24}$ now at University of Kansas, Lawrence, USA

25 also at Max Planck Institute, Munich, Germany, Alexander von Humboldt Research Award

${ }^{26}$ now at KEK, Tsukuba, Japan

27 now at Nagoya University, Japan

${ }^{28}$ Department of Radiological Science, Tokyo Metropolitan University, Japan

${ }^{29}$ now at SunMelx Co. Ltd., Tokyo, Japan

30 PPARC Advanced fellow

31 also at Hamburg University, Inst. of Exp. Physics, Alexander von Humboldt Research Award and partially supported by DESY, Hamburg, Germany

32 also at Łódź University, Poland

33 Łódź University, Poland

${ }^{34}$ now at Lund Universtiy, Lund, Sweden

$\dagger$ deceased 
a supported by the Natural Sciences and Engineering Research Council of Canada (NSERC)

$b$ supported by the German Federal Ministry for Education and Research (BMBF), under contract numbers 05 HZ6PDA, 05 HZ6GUA, 05 HZ6VFA and 05 HZ4KHA

c supported in part by the MINERVA Gesellschaft für Forschung GmbH, the Israel Science Foundation (grant no. 293/02-11.2) and the U.S.-Israel Binational Science Foundation

$d$ supported by the Israel Science Foundation

$e$ supported by the Italian National Institute for Nuclear Physics (INFN)

$f$ supported by the Japanese Ministry of Education, Culture, Sports, Science and Technology (MEXT) and its grants for Scientific Research

$g$ supported by the Korean Ministry of Education and Korea Science and Engineering Foundation

$h \quad$ supported by the Netherlands Foundation for Research on Matter (FOM)

$i$ supported by the Polish State Committee for Scientific Research, project no. DESY/256/2006 - 154/DES/2006/03

$j$ partially supported by the German Federal Ministry for Education and Research $(\mathrm{BMBF})$

$k$ supported by RF Presidential grant N 8122.2006.2 for the leading scientific schools and by the Russian Ministry of Education and Science through its grant for Scientific Research on High Energy Physics

$l$ supported by the Spanish Ministry of Education and Science through funds provided by CICYT

$m$ supported by the Science and Technology Facilities Council, UK

$n$ supported by the US Department of Energy

$o$ supported by the US National Science Foundation. Any opinion, findings and conclusions or recommendations expressed in this material are those of the authors and do not necessarily reflect the views of the National Science Foundation.

$p$ supported by the Polish Ministry of Science and Higher Education as a scientific project (2006-2008)

$q$ supported by FNRS and its associated funds (IISN and FRIA) and by an Inter-University Attraction Poles Programme subsidised by the Belgian Federal Science Policy Office

$r$ supported by the Malaysian Ministry of Science, Technology and Innovation/Akademi Sains Malaysia grant SAGA 66-02-03-0048 


\section{Introduction}

The production of heavy quarks in ep collisions at HERA is an important testing ground for perturbative Quantum Chromodynamics (pQCD) since the large $b$-quark and $c$-quark masses provide a hard scale that allows perturbative calculations. When $Q^{2}$, the negative squared four-momentum exchanged at the electron or positron ${ }^{1}$ vertex, is small, the reactions $e p \rightarrow e b \bar{b} X$ and $e p \rightarrow e c \bar{c} X$ can be considered as a photoproduction process in which a quasi-real photon, emitted by the incoming electron interacts with the proton.

The corresponding leading-order (LO) QCD processes are the direct-photon process, in which the quasi-real photon enters directly in the hard interaction, and the resolvedphoton process, in which the photon acts as a source of partons which take part in the hard interaction. For heavy-quark transverse momenta comparable to the quark mass, next-to-leading-order (NLO) QCD calculations in which the massive quark is generated dynamically $[1,2]$ are expected to provide reliable predictions for the photoproduction cross sections.

Beauty and charm quark production cross sections have been measured using several different methods by both the ZEUS [3-18] and the H1 [19-30] collaborations. Both the deep inelastic scattering (DIS) and photoproduction measurements are reasonably well described by NLO QCD predictions.

Most of the previous measurements of $b$-quark production used muons to tag semileptonic decays of the $B$ hadrons. The identification of electrons close to jets is more difficult than for muons, but the electrons can be identified down to lower momenta. A first measurement of $b$-quark photoproduction from semileptonic decays to electrons $\left(e^{-}\right)$was presented in a previous publication [6], which used the $e^{+} p$ collision data from the 19961997 running period corresponding to an integrated luminosity of $38 \mathrm{pb}^{-1}$. This paper presents an extension of this measurement exploiting semileptonic decays to electrons as well as to positrons for data taken with both $e^{-} p$ and $e^{+} p$ collisions using three times the integrated luminosity. The production of electrons from semileptonic decays $\left(e_{\mathrm{SL}}\right)$, in events with at least two jets $(j j)$ in photoproduction, ep $\rightarrow e b \bar{b} X \rightarrow e j j e_{\mathrm{SL}} X^{\prime}$, was measured in the kinematic range $Q^{2}<1 \mathrm{GeV}^{2}$ and $140 \mathrm{GeV}<W_{\gamma p}<280 \mathrm{GeV}$, where $W_{\gamma p}$ is the centre-of-mass energy of the $\gamma p$ system. The likelihood method used to extract the $b$-quark cross sections also allowed the corresponding $c$-quark cross sections to be extracted. This paper provides a complementary study to the measurements using muon decays.

\footnotetext{
${ }^{1}$ Hereafter unless explicitly stated both electrons and positrons are referred to as electrons.
} 


\section{Experimental set-up}

This analysis was performed with data taken from 1996 to 2000, when HERA collided electrons or positrons with energy $E_{e}=27.5 \mathrm{GeV}$ with protons of energy $E_{p}=820 \mathrm{GeV}$ (1996-1997) or $920 \mathrm{GeV}(1998-2000)$. The corresponding integrated luminosities are 38.6士 $0.6 \mathrm{pb}^{-1}$ at centre-of-mass energy $\sqrt{s}=300 \mathrm{GeV}$, and $81.6 \pm 1.8 \mathrm{pb}^{-1}$ at $\sqrt{s}=318 \mathrm{GeV}$.

A detailed description of the ZEUS detector can be found elsewhere [31]. A brief outline of the components that are most relevant for this analysis is given below.

Charged particles were tracked in the central tracking detector (CTD) [32], which operated in a magnetic field of $1.43 \mathrm{~T}$ provided by a thin superconducting coil. The CTD consisted of 72 cylindrical drift chamber layers, organised in nine superlayers covering the polarangle ${ }^{2}$ region $15^{\circ}<\theta<164^{\circ}$. The transverse-momentum resolution for full-length tracks is $\sigma\left(p_{T}\right) / p_{T}=0.0058 p_{T} \oplus 0.0065 \oplus 0.0014 / p_{T}$, with $p_{T}$ in $\mathrm{GeV}$. The pulse height of the sense wires was read out in order to estimate the ionisation energy loss per unit length, $\mathrm{d} E / \mathrm{d} x$ (see Section 3$)$.

The high-resolution uranium-scintillator calorimeter (CAL) [33] consisted of three parts: the forward (FCAL), the barrel (BCAL) and the rear (RCAL) calorimeters. Each part was subdivided transversely into towers and longitudinally into one electromagnetic section and either one (in RCAL) or two (in BCAL and FCAL) hadronic sections. The smallest subdivision of the calorimeter is called a cell. The CAL energy resolutions, as measured under test-beam conditions, are $\sigma(E) / E=0.18 / \sqrt{E}$ for electrons and $\sigma(E) / E=0.35 / \sqrt{E}$ for hadrons, with $E$ in $\mathrm{GeV}$.

The luminosity was measured from the rate of the bremsstrahlung process ep $\rightarrow$ erp, where the photon was measured in a lead-scintillator calorimeter [34] placed in the HERA tunnel at $Z=-107 \mathrm{~m}$.

\section{$3 \quad \mathrm{~d} E / \mathrm{d} x$ Measurement}

A central tool for this analysis was the $\mathrm{d} E / \mathrm{d} x$ measurement from the CTD. The pulse height of the signals on the sense wires was used to measure the specific ionisation. This pulse height was corrected for a number of effects [35]. Such as a factor $1 / \sin \theta$ due to

\footnotetext{
${ }^{2}$ The ZEUS coordinate system is a right-handed Cartesian system, with the $Z$ axis pointing in the proton beam direction, referred to as the "forward direction", and the $X$ axis pointing left towards the centre of HERA. The coordinate origin is at the nominal interaction point. The pseudorapidity is defined as $\eta=-\ln \left(\tan \frac{\theta}{2}\right)$, where the polar angle, $\theta$, is measured with respect to the proton beam direction. The azimuthal angle, $\phi$, is measured with respect to the $X$ axis.
} 
the projection of the track onto the direction of the signal wire, the space-charge effect caused by the overlap of the ionisation clouds in the avalanche, and the dependence of the pulse shape on the track topology. An additional correction was needed for hits close to the end-plates of the CTD. If a hit followed a previous one on the same wire within $100 \mathrm{~ns}$, its pulse could be distorted: such hits were rejected. The event topology was used to identify additional double hits that could not be resolved; the $\mathrm{d} E / \mathrm{d} x$ measurement was corrected accordingly.

The $\mathrm{d} E / \mathrm{d} x$ value of a track was calculated as the truncated mean value of the individual measurements, corrected as discussed above, after rejecting the lowest $10 \%$ and the highest $30 \%$ of the measurements. Hits where the measured pulse height was in saturation were always rejected in forming the mean. Corrections were applied for the finite number of hits and whenever more than $30 \%$ of the hits were saturated. The corrected $\mathrm{d} E / \mathrm{d} x$ measurement was normalised in units of mip (minimum ionising particles) such that the minimum of the $\mathrm{d} E / \mathrm{d} x$ distribution was 1.0 mip. Electrons are expected to have a mean value of about $1.4 \mathrm{mip}$ in the momentum range studied here.

Different samples of identified particles were used to calibrate and validate the $\mathrm{d} E / \mathrm{d} x$ measurement. The samples used for calibration were:

- $e^{ \pm}$from photon conversions, $J / \psi$ decays and DIS electrons;

- $\pi^{ \pm}$from $K^{0}$ decays with $0.4 \mathrm{GeV}<p<1 \mathrm{GeV}$, where $p$ is the measured track momentum.

The samples used for validation were:

- $\pi^{ \pm}$from $K^{0}$ outside the momentum range used for the calibration sample, as well as $\pi^{ \pm}$from $\rho^{0}, \Lambda$ and $D^{*}$ decays;

- $K^{ \pm}$from $\phi^{0}$ and $D^{*}$ decays;

- $p, \bar{p}$ from $\Lambda$ decays;

- $\operatorname{cosmic} \mu^{ \pm}$.

Typical sample purities were above $99 \%$ for the calibration samples and well above $95 \%$ for the validation samples [35].

After all corrections, the measured $\mathrm{d} E / \mathrm{d} x$ depended only on the particle velocity, $\beta \gamma$. This is illustrated in Fig. 1. It shows the specific energy loss as a function of $\beta \gamma$, for the different samples of identified particles, $e^{ \pm}, \mu^{ \pm}, \pi^{ \pm}, K^{ \pm}, p, \bar{p}$. All particle types are well described using a single physically motivated parametrisation of the mean energy loss as a function of $\beta \gamma$ with five free parameters following Allison and Cobb (AC) [36].

Given the quality of the description of the mean $\mathrm{d} E / \mathrm{d} x$ by the $\mathrm{AC}$ parametrisation, the measurements can be used to determine residuals on $\mathrm{d} E / \mathrm{d} x$. As an example, the 
distribution of residuals for a sample of tracks with the number of hits after truncation, $n_{\text {trunc }}$, equal to 23 is shown in Fig. 2 . The $\mathrm{d} E / \mathrm{d} x$ resolution is typically $11 \%$ for tracks that pass at least five superlayers. It improves to about $9 \%$ for tracks that pass all superlayers.

\section{Monte Carlo simulation}

To evaluate the detector acceptance and to provide the signal and background distributions, Monte Carlo samples of beauty, charm and light-flavour events generated with Pythia 6.2 [37] were used.

The production of $b \bar{b}$-pairs was simulated following the standard PYTHIA prescription with the following subprocesses [38]:

- direct and resolved photoproduction with a leading-order massive matrix element;

- $b$ excitation in both the proton and the photon with a leading-order massless matrix element.

The CTEQ4L [39] parton distributions were used for the proton, while GRV-G LO [40] was used for the photon. The $b$-quark mass parameter was set to $4.75 \mathrm{GeV}$. The production of charm and light quarks was simulated for both direct and non-direct photoproduction with leading-order matrix elements in the massless scheme using the same parton distributions as for the $b \bar{b}$ samples.

The generated events were passed through a full simulation of the ZEUS detector based on Geant 3.13 [41]. The ionisation loss in the CTD was treated separately using a parametrisation of the measured data distributions based on the calibration sample [38, 42]. The final Monte Carlo events had to fulfil the same trigger requirements and pass the same reconstruction programme as the data.

\section{Data selection}

Events were selected online with a three-level trigger $[31,43]$ which required two jets reconstructed in the calorimeter.

The hadronic system (including the decay electron) was reconstructed from energy-flow objects (EFOs) [44] which combine the information from calorimetry and tracking, corrected for energy loss in inactive material. Each EFO was assigned a reconstructed fourmomentum $q^{i}=\left(p_{X}^{i}, p_{Y}^{i}, p_{Z}^{i}, E^{i}\right)$, assuming the pion mass. Jets were reconstructed from EFOs using the $k_{T}$ algorithm [45] in the longitudinally invariant mode with the massive recombination scheme [46] in which $q^{\text {jet }}=\sum_{i} q^{i}$ and the sum runs over all EFOs. The 
transverse energy of the jet was defined as $E_{T}^{\text {jet }}=E^{\text {jet }} \cdot p_{T}^{\text {jet }} / p^{\text {jet }}$, where $E^{\text {jet }}, p^{\text {jet }}$ and $p_{T}^{\text {jet }}$ are the energy, momentum and transverse momentum of the jet, respectively. The transverse energy, $E_{T}^{\mathrm{jet}}$, is therefore always larger than the transverse momentum, $p_{T}^{\mathrm{jet}}$, used in a previous publication [5].

Dijet events were selected as follows:

- at least two jets with $E_{T}^{\text {jet }}>7(6) \mathrm{GeV}$ for the highest (second highest) energetic jet and pseudorapidity of both jets $\left|\eta^{\text {jet }}\right|<2.5$;

- the $Z$ coordinate of the reconstructed primary vertex within $\left|Z_{\mathrm{Vtx}}\right|<50 \mathrm{~cm}$;

- $0.2<y_{\mathrm{JB}}<0.8$, where $y_{\mathrm{JB}}=\left(E-P_{Z}\right) /\left(2 E_{e}\right)$ is the Jacquet-Blondel estimator [47] for the inelasticity, $y$, and $E-P_{Z}=\sum_{i} E^{i}-p_{Z}^{i}$, where the sum runs over all EFOs;

- no scattered-electron candidate found in the calorimeter with energy $E_{e}^{\prime}>5 \mathrm{GeV}$ and $y_{e}<0.9$, with $y_{e}=1-\frac{E_{e}^{\prime}}{2 E_{e}}\left(1-\cos \theta_{e}^{\prime}\right)$, where $\theta_{e}^{\prime}$ is the polar angle of the outgoing electron.

These cuts suppress background from high- $Q^{2}$ events and from non-ep interactions, and correspond to an effective cut of $Q^{2}<1 \mathrm{GeV}^{2}$.

\section{Identification of electrons from semileptonic decays}

Electron candidates were selected among the EFOs by requiring tracks fitted to the primary vertex and having a transverse momentum, $p_{T}^{e}$, of at least $0.9 \mathrm{GeV}$ in the pseudorapidity range $\left|\eta^{e}\right|<1.5$. Only the EFOs consisting of a track matched to a single calorimetric cluster were used. To reduce the hadronic background and improve the overall description, at least $90 \%$ of the EFO energy had to be deposited in the electromagnetic part of the calorimeter. Electron candidates were required to have a track with $n_{\text {trunc }}>12$ to ensure a reliable $\mathrm{d} E / \mathrm{d} x$ measurement. An additional preselection cut of $\mathrm{d} E / \mathrm{d} x>1.1$ mip was applied to reduce the background. Candidates in the angular region corresponding to the gaps between FCAL and BCAL as well as between RCAL and BCAL were removed using a cut on the EFO position [48].

Electrons from photon conversions were tagged and rejected based on the distance of closest approach of a pair of oppositely charged tracks to each other in the plane perpendicular to the beam axis and on their invariant mass [6]. Untagged conversion background and electrons from Dalitz decays were estimated from Monte Carlo studies.

The electron candidate was required to be associated to a jet using the following procedure:

- the jet had to have $E_{T}^{\text {jet }}>6 \mathrm{GeV}$ and $\left|\eta^{\text {jet }}\right|<2.5$; 
- the distance $\Delta R=\sqrt{\left(\eta^{\text {jet }}-\eta^{e}\right)^{2}+\left(\phi^{\text {jet }}-\phi^{e}\right)^{2}}<1.5$;

- in case of more than one candidate jet, the jet closest in $\Delta R$ was chosen.

For the identification of electrons from semileptonic heavy-quark decays, variables for particle identification were combined with event-based information characteristic of heavyquark production. For a given hypothesis of particle, $i$, and source $j$, the likelihood, $\mathcal{L}_{i j}$, is given by

$$
\mathcal{L}_{i j}=\prod_{l} \mathcal{P}_{i j}\left(d_{l}\right)
$$

where $\mathcal{P}_{i j}\left(d_{l}\right)$ is the probability to observe particle $i$ from source $j$ with value $d_{l}$ of a discriminant variable. The particle hypotheses $i \in\{e, \mu, \pi, K, p\}$ and sources, $j$, for electrons from semileptonic beauty, charm decays and background, $j \in\{b, c, \mathrm{Bkg}\}$, were considered. For the likelihood ratio test, the test function, $T_{i j}$ was defined as

$$
T_{i j}=\frac{\alpha_{i} \alpha_{j}^{\prime} \mathcal{L}_{i j}}{\sum_{m, n} \alpha_{m} \alpha_{n}^{\prime} \mathcal{L}_{m n}} .
$$

The $\alpha_{i}, \alpha_{j}^{\prime}$ denote the prior probabilities taken from Monte Carlo. In the sum, $m, n$ run over all particle types and sources defined above. In the following, $T$ is always taken to be the likelihood ratio for an electron originating from a semileptonic $b$-quark decay: $T \equiv T_{e, b}$, unless otherwise stated. The following five discriminant variables were combined in the likelihood test:

- $\mathrm{d} E / \mathrm{d} x$, the average energy loss per unit length of the track in the CTD;

- $E_{\mathrm{EMC}} / E_{\mathrm{CAL}}$, the fraction of the EFO energy taken from the calorimeter information, $E_{\mathrm{CAL}}$, which is deposited in the electromagnetic part, $E_{\mathrm{EMC}}$;

- $E_{\mathrm{CAL}} / p_{\text {track }}$ : the EFO energy divided by the track momentum.

In order to distinguish between electrons from semileptonic $b$-quark and $c$-quark decays and other electron candidates, the following additional observables were used:

- $p_{\perp}^{\text {rel }}$, the transverse-momentum component of the electron candidate relative to the direction of the associated jet defined as

$$
p_{\perp}^{\mathrm{rel}}=\frac{\left|\vec{p}_{\mathrm{jet}} \times \vec{p}_{e}\right|}{\left|\vec{p}_{\mathrm{jet}}\right|}
$$

where $\vec{p}_{e}$ is the momentum of the electron candidate. The variable $p_{\perp}^{\text {rel }}$ can be used to discriminate between electrons from semileptonic $b$-quark decays and from other sources, because its distribution depends on the mass of the decaying particle. It is not possible to distinguish charm from light-flavour decays with this variable; 
- $\Delta \phi$, the difference of azimuthal angles of the electron candidate and the missing transverse momentum vector defined as

$$
\Delta \phi=\left|\phi\left(\vec{p}_{e}\right)-\phi\left(\vec{p}_{T}\right)\right|
$$

where $\vec{p}_{T}$ is the negative vector sum of the EFO momentum transverse to the beam axis,

$$
\vec{p}_{T}=-\left(\sum_{i} p_{x}^{i}, \sum_{i} p_{y}^{i}\right)
$$

and the sum runs over all EFOs. The vector $\vec{p}_{T}$ is used as an estimator of the direction of the neutrino from the semileptonic decay. The variable $\Delta \phi$ can be used to discriminate semileptonic decays of $b$ quarks and $c$ quarks from other sources.

The shapes of the charm- and light-quark $p_{\perp}^{\text {rel }}$ distributions in the Monte Carlo were corrected [5] using a dedicated background sample in the data. The value of the correction increased with $p_{\perp}^{\text {rel }}$ and was $15 \%$ at $p_{\perp}^{\text {rel }}=1.5 \mathrm{GeV}$, where the purity of the $b$ contribution is highest. For the $\Delta \phi$ distribution a correction was determined in a similar way, but in this case the maximal correction was only of the order of $5 \%$.

In Fig. 3 the distributions of the five input variables used in the likelihood are shown for electrons from $b$-quark and $c$-quark decays and for electron candidates from other sources. A clear difference in shape between signal and background can be seen.

\section{Signal extraction}

The electron candidates in the Monte Carlo samples were classified as originating from beauty, charm or background. The beauty sample also contains the cascade decays $b \rightarrow$ $c \rightarrow e$, but not $b \rightarrow \tau \rightarrow e$ and $b \rightarrow J / \psi \rightarrow e^{+} e^{-}$that have been included in the background sample. Test functions (see Section 6) were calculated separately for the three samples. The fractions of the three samples in the data, $f_{e, b}^{\mathrm{DATA}}, f_{e, c}^{\mathrm{DATA}}, f_{\mathrm{Bkg}}^{\mathrm{DATA}}$, were obtained from a three-component maximum-likelihood fit [49] to the $T$ distributions. The constraint $f_{e, b}^{\mathrm{DATA}}+f_{e, c}^{\mathrm{DATA}}+f_{\mathrm{Bkg}}^{\mathrm{DATA}}=1$ was imposed in the fit. The fit range of the test function was restricted to $-2 \ln T<10$ to remove the region dominated by background and where the test function falls rapidly. The $\chi^{2}$ for the fit is $\chi^{2} / \mathrm{ndf}=13 / 12$ and the $b$-quark and $c$-quark measurements have a correlation coefficient of -0.6 . The result of the fit is shown in Fig. 4 and corresponds to a scaling of the cross section predicted by the beauty Monte Carlo by a factor of $1.75 \pm 0.16$ and the charm Monte Carlo by a factor of $1.28 \pm 0.13$. These factors are applied to Figs. 5-8 and denoted as "PYTHIA (scaled)". A fit over the whole $T$ range gave consistent cross sections and was used as a cross-check. The distributions of the five variables that entered the likelihood are shown in Fig. 5. The description of all variables is reasonable. These distributions are dominated by the 
background contribution. In order to select a beauty-enriched sample, a cut of $-2 \ln T<1$ was applied. The resulting distributions are shown in Fig. 6. A likelihood for semileptonic charm can also be constructed, $T_{e, c}$. The distributions of the likelihood for a sample satisfying $-2 \ln T_{e, c}<1.5$ are shown in Fig. 7. Good agreement is observed in both cases. To demonstrate the quality of the data description by the Monte Carlo, the distributions of $E_{T}^{\text {jet }}$ and $\eta^{\text {jet }}$ of the jet associated with the electron and of the $p_{T}^{e}$ of the electron candidates are compared in Fig. 8a)-c). In Fig. 8d)-i) the same distributions are compared for the beauty- and charm enriched-samples. Some differences are observed in the jet variables, mainly in the region dominated by background. The agreement significantly improves for samples enriched in beauty and charm signal.

\section{Cross section determination}

The cross sections have been measured in the kinematic range $Q^{2}<1 \mathrm{GeV}^{2}, 0.2<y<0.8$, with at least two jets with $E_{T}^{\text {jet }}>7(6) \mathrm{GeV},\left|\eta^{\text {jet }}\right|<2.5$ and an electron from a semileptonic decay with $p_{T}^{e}>0.9 \mathrm{GeV}$ in the range $\left|\eta^{e}\right|<1.5$.

The differential beauty cross section for a variable, $v$, was determined separately for each bin, $k$, from the relative fractions in the data obtained from the fit and the acceptance correction, $\mathcal{A}_{v_{k}}^{b}$, calculated using the Monte Carlo,

$$
\frac{d \sigma_{b}}{d v_{k}}=\frac{N^{\mathrm{DATA}} \cdot f_{e, b}^{\mathrm{DATA}}\left(v_{k}\right)}{\mathcal{A}_{v_{k}}^{b} \cdot \mathcal{L} \cdot \Delta v_{k}}
$$

where $N^{\text {DATA }}$ is the number of electron candidates found in the data, $\mathcal{L}$ is the integrated luminosity and $\Delta v_{k}$ is the bin width.

In order to determine the acceptance, the jet-finding algorithm was applied to the MC events after the detector simulation and at hadron level. The acceptance is defined as

$$
\mathcal{A}=\frac{N_{e}^{\text {obs }}}{N_{e}^{\text {had }}}
$$

where $N_{e}^{\text {obs }}$ is the number of electrons from semileptonic decays reconstructed in the Monte Carlo satisfying the selection criteria detailed in Sections 5 and 6 , and $N_{e}^{\text {had }}$ is the number of electrons from semileptonic decays produced in the signal process that satisfy the kinematic requirements using the Monte Carlo information at the generator level. At hadron level, the $k_{T}$ algorithm was applied to all final-state particles with a lifetime of $\tau>0.01 \mathrm{~ns}$ and the electron was associated to its parent jet using the generator information. 
All cross sections were measured separately for the two centre-of-mass energies $\sqrt{s}=300$ and $318 \mathrm{GeV}$. Additionally, the cross sections were calculated with the whole data set and were corrected to $\sqrt{s}=318 \mathrm{GeV}$. The correction factor of $\approx 2 \%$ was determined with LO as well as NLO calculations.

The charm cross sections were measured using the same procedure.

\section{Systematic uncertainties}

The systematic uncertainties were calculated by varying the analysis procedure and then redoing the fit to the likelihood distributions. The following sources were the main contributors to the systematic uncertainty (the first value in parentheses is the uncertainty for beauty, while the second is that for charm):

- the systematic uncertainty on the description of the $\mathrm{d} E / \mathrm{d} x$ information was estimated by looking at the differences between the various calibration and validation samples. Variations in the mean, width and shape of the distributions were evaluated and used as a measure of the uncertainty [35]. The resulting uncertainty was found to be $\left(_{-5}^{+1} \%\right.$ $/{ }_{-3}^{+10 \%)}$

- the changes in the correction to the $p_{\perp}^{\text {rel }}$ distribution in various kinematic ranges were taken as a measure of its uncertainty. For $p_{\perp}^{\mathrm{rel}}=1.5 \mathrm{GeV}$ the variation was $20 \%$ of the correction. The changes led to a systematic uncertainty of $\left({ }_{-6}^{+3} \% /{ }_{-5}^{+10} \%\right)$.

In addition, the correction to the charm distribution was varied from zero to that of the background sample. This led to an uncertainty of $\left({ }_{-4}^{+6} \% /{ }_{-1}^{+7} \%\right)$;

- a shift of the CAL energy scale in the Monte Carlo simulation by $\pm 3 \%( \pm 2 \% / \pm 5 \%)$;

- reweighting of the direct and non-direct contributions in the Monte Carlo to provide a better description of the data $(+1 \% /+3 \%)$;

- the estimated residual number of electrons left in the sample from photon conversions as well as from Dalitz decays were varied by $25 \%$ and $20 \%$ respectively [50]. This led to systematic uncertainties of $( \pm 1 \% / \mp 4 \%)$ due to photon conversions and $( \pm 1 \% /$ $\mp 1 \%$ ) due to Dalitz decays.

These systematic uncertainties were added in quadrature separately for the negative and the positive variations to determine the overall systematic uncertainty of ${ }_{-9}^{+8} \%$ for the beauty and ${ }_{-9}^{+17 \%}$ for the charm cross sections. Since no significant dependence of the systematic uncertainties on the kinematic variables was observed, the same uncertainty was applied to each data point. A $2 \%$ overall normalisation uncertainty associated with the luminosity measurement was included. 
A series of further checks were made. The cut on the transverse momentum of the electron candidate was varied by $\pm 3 \%$, which is the momentum uncertainty for a track with $p_{T}=0.9 \mathrm{GeV}$. The $\Delta \phi$ correction was varied within its uncertainty. The cut on $\Delta R$ to associate the decay electron with a jet was varied between 1.5 and 1.0. The effect of the gaps between FCAL and BCAL as well as between RCAL and BCAL was investigated by varying the cut on the EFO position. Various tests of the signal-extraction method were made: e.g. using the likelihood without the $E_{\mathrm{EMC}} / E_{\mathrm{CAL}}$ or $E_{\mathrm{CAL}} / p_{\text {track }}$ variables; applying the fit on a signal-enriched sample by making tighter cuts on the input variables and varying the fit range. The prior probabilities were recalculated after the fit and used as the input for a second fit iteration. Separate fits were made for electron and positron candidates for each of the lepton beam particles $\left(e^{-}\right.$and $\left.e^{+}\right)$separately as well as for the combined sample. All variations were found to be consistent with the expected fluctuations due to statistics; therefore they have not been added to the systematic uncertainty.

\section{Theoretical predictions and uncertainties}

QCD predictions at NLO, based on the FMNR programme [51], are compared to the data. The programme separately generates processes containing point-like and hadronlike photon contributions, which have to be combined to obtain the total cross section. The $b \bar{b}$ and the $c \bar{c}$ production cross sections were calculated separately. The parton distribution functions were taken from CTEQ5M [52] for the proton and GRV-G HO [40] for the photon. The heavy-quark masses (pole masses) were set to $m_{b}=4.75 \mathrm{GeV}$ and $m_{c}=1.6 \mathrm{GeV}$. The strong coupling constant, $\Lambda_{\mathrm{QCD}}^{(5)}$, was set to $0.226 \mathrm{GeV}$. The renormalisation, $\mu_{R}$, and factorisation, $\mu_{F}$, scales were chosen to be equal and set to $\mu_{R}=\mu_{F}=\sqrt{\hat{p}_{T}^{2}+m_{b(c)}^{2}}$, where $\hat{p}_{T}$ is the average transverse momentum of the heavy quarks.

The Peterson fragmentation function [53], with $\epsilon_{b}=0.0035$ and $\epsilon_{c}=0.035$ [54], was used to produce beauty and charm hadrons from the heavy quarks. For the $b \bar{b}$ and $c \bar{c}$ cross sections, the decays into electrons were simulated using decay spectra from PYTHIA.

For beauty, both the contributions from prompt and from cascade decays, excluding $b \rightarrow \tau \rightarrow e$ and $b \rightarrow J / \psi \rightarrow e^{+} e^{-}$, are taken into account in the effective branching fraction. The values were set to 0.221 for the $b \bar{b}$ and to 0.096 for the $c \bar{c}$ cross sections [55].

For the systematic uncertainty on the theoretical prediction, the masses and scales were varied simultaneously to maximise the change in the cross section using the values: $m_{b}=4.5,5.0 \mathrm{GeV}, m_{c}=1.35,1.85 \mathrm{GeV}$ and $\mu_{R}=\mu_{F}=\frac{1}{2} \sqrt{\hat{p}_{T}^{2}+m_{b(c)}^{2}}, 2 \sqrt{\hat{p}_{T}^{2}+m_{b(c)}^{2}}$. 
The effects of different parton density functions as well as variations of $\epsilon_{b}$ within the uncertainty of 0.0015 had a small effect on the cross-section predictions and were neglected. The parameter $\epsilon_{c}$ was varied between 0.02 and 0.07 and the contribution was added in quadrature to the systematic uncertainty. The uncertainty on the electron decay spectra, evaluated from comparisons to experimental measurements $[56,57]$ and to a simple freequark decay model, was found to be small compared to the total theoretical uncertainty and was neglected.

The uncertainty on the NLO QCD predictions for the total cross section are $+25 \%$ and $-15 \%$ for beauty and $+45 \%$ and $-28 \%$ for charm.

The NLO QCD predictions for parton-level jets, reconstructed by applying the $k_{T}$ algorithm to the outgoing partons, were corrected for hadronisation effects. A bin-by-bin procedure was used according to $d \sigma=d \sigma_{\mathrm{NLO}} \cdot C^{\text {had }}$, where $d \sigma_{\mathrm{NLO}}$ is the cross section for partons in the final state of the NLO calculation. The hadronisation correction factor, $C^{\text {had }}$, was defined as the ratio of the dijet cross sections, extracted from the PYTHIA Monte Carlo, after and before the hadronisation process, $C^{\text {had }}=d \sigma_{\mathrm{MC}}^{\text {Hadrons }} / d \sigma_{\mathrm{MC}}^{\text {Partons }}$. The hadronic corrections were generally small and are given in Tables 1-5. No uncertainty was assigned to the correction.

\section{Results}

The visible $e p$ cross sections (quoted at hadron level) for b-quark and $c$-quark production and the subsequent semileptonic decay to an electron with $p_{T}^{e}>0.9 \mathrm{GeV}$ in the range $\left|\eta^{e}\right|<1.5$ in photoproduction events with $Q^{2}<1 \mathrm{GeV}^{2}$ and $0.2<y<0.8$ and at least two jets with $E_{T}>7(6) \mathrm{GeV},|\eta|<2.5$ were determined separately for $\sqrt{s}=300 \mathrm{GeV}$ and $\sqrt{s}=318 \mathrm{GeV}$. The measurements are given in Table 1 and are shown in Fig. 9. The cross sections at the two different centre-of-mass energies are consistent with each other; combining the results leads to a reduced statistical uncertainty. For the complete data set $(96-00)$ the cross sections evaluated at $\sqrt{s}=318 \mathrm{GeV}$ are

$$
\begin{aligned}
& \left.\left.\sigma_{b}^{\text {vis }}=(125 \pm 11 \text { (stat. })_{-11}^{+10} \text { (syst. }\right)\right) \mathrm{pb}, \\
& \left.\sigma_{c}^{\text {vis }}=\left(278 \pm 33(\text { stat. })_{-24}^{+48} \text { (syst. }\right)\right) \mathrm{pb} .
\end{aligned}
$$

The visible $b$-quark cross section was also determined in the kinematic region of a previous ZEUS analysis using muons [5] and is in good agreement.

The NLO QCD predictions of FMNR (see Table 1) are compared to the data in Fig. 9. Good agreement is observed. Also shown in Fig. 9 are expectations of the Pythia Monte Carlo. The combined $b(\bar{b})$ cross section is a factor 1.75 higher while the $c(\bar{c})$ cross section 
is a factor of 1.28 higher than the PYTHIA prediction (see Section 7). These factors are used to scale the PYTHIA predictions in the following figures.

Differential cross sections as a function of $p_{T}^{e}$ and $\eta_{e}, x_{\gamma}^{\text {obs }}, E_{T}^{\text {jet } 1}$ and $\eta^{\text {jet } 1}$ are shown in Figs. 10, 11 and 12, respectively. The variable $x_{\gamma}^{\text {obs }}$ is defined as

$$
x_{\gamma}^{\mathrm{obs}}=\frac{\sum_{i=1,2}\left(E^{\mathrm{jet} i}-p_{Z}^{\mathrm{jet} i}\right)}{E-p_{Z}},
$$

where the sum is over the two highest-energy jets, and corresponds at leading order to the fraction of the exchanged-photon momentum in the hard scattering process. The figures also show the NLO QCD and the scaled PYTHIA predictions. The cross-section values are given in Tables 2-4. Both the predictions from the NLO QCD calculations as well as the scaled Pythia cross sections describe the data well.

The differential cross sections as a function of the transverse energy of the jet associated with the electron from the semileptonic decay, $E_{T}^{\text {e jet }}$, were also determined. These cross sections are shown in Fig. 13 and given in Table 5. The good agreement with the NLO QCD prediction allows the cross section as a function of $p_{T}^{b}$ to be extracted [6]. The resulting cross section is shown in Fig. 14 and is also compared with previous ZEUS measurements $[3,5,6]$. The results presented here overlap in $p_{T}^{b}$ with these previous measurements and have comparable or smaller uncertainties, giving a consistent picture of $b$-quark production in ep collisions in the photoproduction regime.

\section{Conclusions}

Beauty and charm production have been measured in dijet photoproduction using semileptonic decays into electrons. The results were obtained by simultaneously extracting the $b$ - and $c$-quark cross sections using a likelihood ratio optimised for $b$-quark production. One of the most important variables in the likelihood was the $\mathrm{d} E / \mathrm{d} x$ measurement in the central tracking detector.

The results were compared to both NLO QCD calculations as well as predictions from Monte Carlo models. The NLO QCD predictions are consistent with the data. The Monte Carlo models describe well the shape of the differential distributions in the data. The results on $b$-quark production are also in agreement with a previous less precise ZEUS measurement using semileptonic decays into electrons. Within the momentum range covered by previous ZEUS measurements using decays into muons, good agreement is found.

The cross section as a function of the transverse momentum of the $b$ quarks has been measured over a wide range. The measurements agree well with the previous values, 
giving a consistent picture of $b$-quark production in $e p$ collisions in the photoproduction regime, and are well reproduced by the NLO QCD calculations.

\section{Acknowledgements}

We thank the DESY Directorate for their strong support and encouragement. The remarkable achievements of the HERA machine group were essential for the successful completion of this work. The design, construction and installation of the ZEUS detector have been made possible by the effort of many people who are not listed as authors. 


\section{References}

[1] M. Glück, E. Reya and A. Vogt, Phys. Rev. D 45, 3986 (1992).

[2] S. Frixione, P. Nason and G. Ridolfi, Nucl. Phys. B 454 (1995);

S. Frixione et al., Phys. Lett. B 348, 633 (1995).

[3] ZEUS Collab., S. Chekanov et al., Eur. Phys. J. C 50, 1434 (2007).

[4] ZEUS Collab., S. Chekanov et al., Phys. Lett. B 599, 173 (2004).

[5] ZEUS Collab., S. Chekanov et al., Phys. Rev. D 70, 12008 (2004). Erratum-ibid D 74, 59906 (2006).

[6] ZEUS Collab., J. Breitweg et al., Eur. Phys. J. C 18, 625 (2001).

[7] ZEUS Collab., S. Chekanov et al., Phys. Lett. B 649, 111 (2007).

[8] ZEUS Collab., S. Chekanov et al., Preprint DESY-07-52 (arXiv:0704.3562v1 [hep-ex]), 2007. Accepted by JHEP.

[9] ZEUS Collab., S. Chekanov et al., Nucl. Phys. B 729, 492 (2005).

[10] ZEUS Collab., S. Chekanov et al., Eur. Phys. J. C 44, 351 (2005).

[11] ZEUS Collab., S. Chekanov et al., Phys. Rev. D 69, 012004 (2004).

[12] ZEUS Collab., S. Chekanov et al., Phys. Lett. B 565, 87 (2003).

[13] ZEUS Collab., J. Breitweg et al., Eur. Phys. J. C 12, 35 (2000).

[14] ZEUS Collab., J. Breitweg et al., Phys. Lett. B 481, 213 (2000).

[15] ZEUS Collab., J. Breitweg et al., Eur. Phys. J. C 6, 67 (1999).

[16] ZEUS Collab., J. Breitweg et al., Phys. Lett. B 401, 192 (1997).

[17] ZEUS Collab., J. Breitweg et al., Phys. Lett. B 407, 402 (1997).

[18] ZEUS Collab., M. Derrick et al., Phys. Lett. B 349, 225 (1995).

[19] H1 Collab., A. Aktas et al., Eur. Phys. J. C 47, 597 (2006).

[20] H1 Collab., A. Aktas et al., Eur. Phys. J. C 45, 23 (2006).

[21] H1 Collab., A. Aktas et al., Phys. Lett. B 621, 56 (2005).

[22] H1 Collab., A. Aktas et al., Eur. Phys. J. C 40, 349 (2005).

[23] H1 Collab., A. Aktas et al., Eur. Phys. J. C 41, 453 (2005).

[24] H1 Collab., C. Adloff et al., Phys. Lett. B 467, 156 (1999).

[25] H1 Collab., A. Aktas et al., Eur. Phys. J. C 51, 271 (2007).

[26] H1 Collab., A. Aktas et al., Eur. Phys. J. C 50, 251 (2006). 
[27] H1 Collab., A. Aktas et al., Eur. Phys. J. C 38, 447 (2005).

[28] H1 Collab., C. Adloff et al., Phys. Lett. B 528, 199 (2002).

[29] H1 Collab., C. Adloff et al., Nucl. Phys. B 545, 21 (1999).

[30] H1 Collab., S. Aid et al., Nucl. Phys. B 472, 32 (1996).

[31] ZEUS Collab., U. Holm (ed.), The ZEUS Detector. Status Report (unpublished), DESY (1993), available on http://www-zeus.desy.de/bluebook/bluebook.html.

[32] N. Harnew et al., Nucl. Instr. and Meth. A 279, 290 (1989);

B. Foster et al., Nucl. Phys. Proc. Suppl. B 32, 181 (1993);

B. Foster et al., Nucl. Instr. and Meth. A 338, 254 (1994).

[33] M. Derrick et al., Nucl. Instr. and Meth. A 309, 77 (1991);

A. Andresen et al., Nucl. Instr. and Meth. A 309, 101 (1991);

A. Caldwell et al., Nucl. Instr. and Meth. A 321, 356 (1992);

A. Bernstein et al., Nucl. Instr. and Meth. A 336, 23 (1993).

[34] J. Andruszków et al., Preprint DESY-92-066, DESY, 1992;

ZEUS Collab., M. Derrick et al., Z. Phys. C 63, 391 (1994);

J. Andruszków et al., Acta Phys. Pol. B 32, 2025 (2001).

[35] D. Bartsch, Energy-loss measurement with the ZEUS Central Tracking Detector. Ph.D. Thesis, Universität Bonn, Bonn, Germany, Report BONN-IR-2007-05, 2007, available on http://www-zeus.physik.uni-bonn.de/german/phd.html.

[36] W.W.M. Allison and J.H. Cobb, Annual Review in Nuclear \& Particle Physics 30, 253 (1980).

[37] T. Sjöstrand et al., Comp. Phys. Comm. 135, 238 (2001);

E. Norrbin and T. Sjöstrand, Eur. Phys. J. C 17, 137 (2000);

T. Sjöstrand, L. Lönnblad, and S. Mrenna, Preprint hep-ph/0108264, 2001.

[38] O.M. Kind, Production of Heavy Flavours with Associated Jets at HERA. Ph.D. Thesis, Universität Bonn, Bonn, Germany, Report BONN-IR-2007-04, 2007, available on http://www-zeus.physik.uni-bonn.de/german/phd.html.

[39] H.L. Lai et al., Phys. Rev. D 55, 1280 (1997).

[40] M. Glück, E. Reya and A. Vogt, Phys. Rev. D 46, 1973 (1992).

[41] R. Brun et al., GeAnt3, Technical Report CERN-DD/EE/84-1, CERN, 1987.

[42] R. Zimmermann, Kalibrierung und Charakterisierung der dE/dx-Information der Zentralen Driftkammer bei ZEUS. Diploma Thesis, Universität Bonn, Bonn, Germany, Report BONN-IB-2007-10, 2007, available on http: //www-zeus . physik . uni-bonn. de/german/diploma.html. 
[43] W.H. Smith, K. Tokushuku and L.W. Wiggers, Proc. Computing in High-Energy Physics (CHEP), Annecy, France, C. Verkerk and W. Wojcik (eds.), p. 222. CERN, Geneva, Switzerland (1992). Also in preprint DESY 92-150B.

[44] ZEUS Collab., J. Breitweg et al., Eur. Phys. J. C 1, 81 (1998); G.M. Briskin, Diffractive Dissociation in ep Deep Inelastic Scattering. Ph.D. Thesis, Tel Aviv University, Report DESY-THESIS 1998-036, 1998.

[45] S.D. Ellis and D.E. Soper, Phys. Rev. D 48, 3160 (1993).

[46] S. Catani et al., Nucl. Phys. B 406, 187 (1993).

[47] F. Jacquet and A. Blondel, Proceedings of the Study for an ep Facility for Europe, U. Amaldi (ed.), p. 391. Hamburg, Germany (1979). Also in preprint DESY 79/48.

[48] M. Jüngst, Elektronidentifikation mit dem ZEUS-Detektor und Bestimmung des Beauty-Produktionsquerschnitts. Diploma Thesis, Universität Bonn, Bonn, Germany, Report BONN-IB-05-15, 2005, available on http://www-zeus.physik. uni-bonn.de/german/diploma.html.

[49] R. Barlow and C. Beeston, Comp. Phys. Comm. 77, 219 (1993).

[50] W. Verkerke, Measurement of Charm Production Deep Inelastic Scattering. Ph.D. Thesis, University of Amsterdam, 1998. Unpublished.

[51] S. Frixione et al., Nucl. Phys. B 412, 225 (1994);

S. Frixione et al., Adv. Ser. Direct. High Energy Phys. 15, 609 (1998).

[52] CTEQ Collab., H.L. Lai et al., Eur. Phys. J. C 12, 375 (2000).

[53] C. Peterson et al., Phys. Rev. D 27, 105 (1983).

[54] P. Nason and C. Oleari, Nucl. Phys. B 565, 245 (2000).

[55] Particle Data Group, W.-M. Yao et al., J. Phys G 33, 1 (2006).

[56] BELLE Collaboration, K. Abe et al., Phys. Lett. B 547, 181 (2002); BABAR Collaboration, B. Auert et al., Phys. Rev. D 67, 031101 (2003).

[57] CLEO Collaboration, N.E. Adam et al., Phys. Rev. Lett. 97, 251801 (2006). 


\begin{tabular}{c|ccc|ccc} 
& $\begin{array}{c}\sigma_{b}^{\mathrm{vis}} \\
(\mathrm{pb})\end{array}$ & $\begin{array}{c}\sigma_{b}^{\mathrm{NLO}} \\
(\mathrm{pb})\end{array}$ & $C_{b}^{\mathrm{had}}$ & $\begin{array}{c}\sigma_{c}^{\mathrm{vis}} \\
(\mathrm{pb})\end{array}$ & $\begin{array}{c}\sigma_{c}^{\mathrm{NLO}} \\
(\mathrm{pb})\end{array}$ & $C_{c}^{\mathrm{had}}$ \\
\hline $96-97$ & $101 \pm 18_{-9}^{+8}$ & $81_{-12}^{+20}$ & 0.81 & $253 \pm 58_{-22}^{+44}$ & $360_{-100}^{+160}$ & 1.00 \\
$98-00$ & $139 \pm 16_{-12}^{+11}$ & $88_{-13}^{+22}$ & 0.81 & $260 \pm 40_{-23}^{+45}$ & $380_{-110}^{+170}$ & 1.01 \\
\hline $96-00$ & $125 \pm 11_{-11}^{+10}$ & $88_{-13}^{+22}$ & 0.81 & $278 \pm 33_{-24}^{+48}$ & $380_{-110}^{+170}$ & 1.01
\end{tabular}

Table 1: Total cross sections for electrons from $b$ or $c$ quarks in photoproduction events, $Q^{2}<1 \mathrm{GeV}^{2}$ and $0.2<y<0.8$, with at least two jets with $E_{T}^{\text {jet }}>7(6) \mathrm{GeV}$, $\left|\eta^{\text {jet }}\right|<2.5$ and the subsequent semileptonic decay to an electron with $p_{T}^{e}>0.9 \mathrm{GeV}$ and $\left|\eta^{e}\right|<1.5$. The values are given separately for $\sqrt{s}=300 \mathrm{GeV}$ (96-97) and $\sqrt{s}=318 \mathrm{GeV}$ (98-00) as well as for the complete data set (96-00) extrapolated to $\sqrt{s}=318 \mathrm{GeV}$. The first error is statistical and the second is systematic. In addition, the NLO QCD prediction and its uncertainty is given, after applying the appropriate hadronisation correction $\left(C_{b}^{\mathrm{had}}, C_{c}^{\mathrm{had}}\right)$.

\begin{tabular}{c|ccc|ccc}
$\begin{array}{c}p_{T}^{e} \\
(\mathrm{GeV})\end{array}$ & $\begin{array}{c}d \sigma_{b} / d p_{T}^{e} \\
(\mathrm{pb} / \mathrm{GeV})\end{array}$ & $\begin{array}{c}d \sigma_{b}^{\mathrm{NLO}} / d p_{T}^{e} \\
(\mathrm{pb} / \mathrm{GeV})\end{array}$ & $C_{b}^{\mathrm{had}}$ & $\begin{array}{c}d \sigma_{c} / d p_{T}^{e} \\
(\mathrm{pb} / \mathrm{GeV})\end{array}$ & $\begin{array}{c}d \sigma_{c}^{\mathrm{NLO}} / d p_{T}^{e} \\
(\mathrm{pb} / \mathrm{GeV})\end{array}$ & $C_{c}^{\text {had }}$ \\
\hline $0.9: 2.1$ & $56.3 \pm 9.6_{-5.0}^{+4.3}$ & $34_{-7}^{+11}$ & 0.78 & $117 \pm 26_{-10}^{+20}$ & $177_{-38}^{+71}$ & 1.02 \\
$2.1: 3.3$ & $24.0 \pm 3.7_{-2.1}^{+1.8}$ & $16.8_{-3.5}^{+5.9}$ & 0.79 & $54.4 \pm 9.0_{-4.8}^{+9.5}$ & $80_{-23}^{+42}$ & 0.98 \\
$3.3: 4.5$ & $11.9 \pm 2.6_{-1.1}^{+0.9}$ & $9.9_{-2.3}^{+3.6}$ & 0.84 & $26.0 \pm 5.8_{-2.3}^{+4.5}$ & $36_{-14}^{+27}$ & 0.99 \\
$4.5: 8.0$ & $4.7 \pm 1.9_{-0.4}^{+0.4}$ & $3.3_{-0.9}^{+1.4}$ & 0.94 & $1.5 \pm 2.7_{-0.1}^{+0.3}$ & $7.5_{-4.0}^{+9.5}$ & 0.99 \\
& \multicolumn{7}{|c|}{} & & \\
$\eta^{e}$ & $d \sigma_{b} / d \eta^{e}$ & $d \sigma_{b}^{\mathrm{NLO}} / d \eta^{e}$ & $C_{b}^{\mathrm{had}}$ & $d \sigma_{c} / d \eta^{e}$ & $d \sigma_{c}^{\mathrm{NLO}} / d \eta^{e}$ & $C_{c}^{\text {had }}$ \\
& $(\mathrm{pb})$ & $(\mathrm{pb})$ & & $(\mathrm{pb})$ & $(\mathrm{pb})$ & \\
\hline$-1.5:-0.5$ & $26.4 \pm 4.6_{-2.4}^{+2.0}$ & $16.7_{-3.6}^{+6.6}$ & 0.75 & $51 \pm 12_{-4}^{+9}$ & $111_{-33}^{+66}$ & 0.98 \\
$-0.5: 0.0$ & $53.4 \pm 9.1_{-4.8}^{+4.1}$ & $39.5_{-8.3}^{+13.8}$ & 0.81 & $152 \pm 25_{-13}^{+26}$ & $192_{-53}^{+100}$ & 1.01 \\
$0.0: 0.5$ & $57.7 \pm 11.6_{-5.1}^{+4.4}$ & $41.9_{-9.0}^{+13.9}$ & 0.82 & $187 \pm 36_{-16}^{+33}$ & $165_{-43}^{+82}$ & 1.02 \\
$0.5: 1.5$ & $42.4 \pm 8.7_{-3.8}^{+3.2}$ & $28.1_{-6.3}^{+10.1}$ & 0.84 & $36 \pm 24_{-3}^{+6}$ & $90_{-26}^{+51}$ & 1.02
\end{tabular}

Table 2: Differential electron cross sections as a function of $p_{T}^{e}$ and $\eta^{e}$ for the complete data set. For further details see the caption of Table 1.

\begin{tabular}{c|ccc|ccc}
$x_{\gamma}^{\mathrm{obs}}$ & $\begin{array}{c}d \sigma_{b} / d x_{\gamma}^{\mathrm{obs}} \\
(\mathrm{pb})\end{array}$ & $\begin{array}{c}d \sigma_{b}^{\mathrm{NLO}} / d x_{\gamma}^{\mathrm{obs}} \\
(\mathrm{pb})\end{array}$ & $C_{b}^{\mathrm{had}}$ & $\begin{array}{c}d \sigma_{c} / d x_{\gamma}^{\mathrm{obs}} \\
(\mathrm{pb})\end{array}$ & $\begin{array}{c}d \sigma_{c}^{\mathrm{NLO}} / d x_{\gamma}^{\mathrm{obs}} \\
(\mathrm{pb})\end{array}$ & $C_{c}^{\mathrm{had}}$ \\
\hline $0.00: 0.45$ & $51 \pm 17_{-5}^{+4}$ & $28_{-10}^{+18}$ & 1.07 & $70 \pm 35_{-6}^{+12}$ & $122_{-56}^{+108}$ & 1.16 \\
$0.45: 0.75$ & $166 \pm 25_{-15}^{+13}$ & $81_{-28}^{+50}$ & 2.27 & $227 \pm 49_{-20}^{+40}$ & $216_{-85}^{+178}$ & 1.32 \\
$0.75: 1.00$ & $216 \pm 31_{-19}^{+17}$ & $166_{-30}^{+47}$ & 0.55 & $715 \pm 79_{-63}^{+124}$ & $920_{-190}^{+370}$ & 0.90
\end{tabular}

Table 3: Differential cross sections as a function of $x_{\gamma}^{o b s}$ for the complete data set. For further details see the caption of Table 1. 


\begin{tabular}{c|ccc|ccc}
$\begin{array}{c}E_{T}^{\text {jet } 1} \\
(\mathrm{GeV})\end{array}$ & $\begin{array}{c}d \sigma_{b} / E_{T}^{\text {jet } 1} \\
(\mathrm{pb} / \mathrm{GeV})\end{array}$ & $\begin{array}{c}d \sigma_{b}^{\mathrm{NLO}} / E_{T}^{\text {jet } 1} \\
(\mathrm{pb} / \mathrm{GeV})\end{array}$ & $C_{b}^{\mathrm{had}}$ & $\begin{array}{c}d \sigma_{c} / E_{T}^{\text {jet } 1} \\
(\mathrm{pb} / \mathrm{GeV})\end{array}$ & $\begin{array}{c}d \sigma_{c}^{\mathrm{NLO}} / E_{T}^{\text {jet } 1} \\
(\mathrm{pb} / \mathrm{GeV})\end{array}$ & $C_{c}^{\text {had }}$ \\
\hline $7: 10$ & $16.8 \pm 2.5_{-1.5}^{+1.3}$ & $10.1_{-1.9}^{+3.2}$ & 0.59 & $45.9 \pm 7.3_{-4.0}^{+8.0}$ & $72_{-19}^{+43}$ & 0.99 \\
$10: 13$ & $12.0 \pm 1.9_{-1.1}^{+0.9}$ & $9.4_{-2.3}^{+3.7}$ & 0.97 & $28.0 \pm 4.7_{-2.4}^{+4.9}$ & $35_{-12}^{+14}$ & 1.07 \\
$13: 16$ & $8.3 \pm 1.6_{-0.7}^{+0.6}$ & $5.1_{-1.1}^{+2.0}$ & 1.18 & $5.9 \pm 3.4_{-0.5}^{+1.0}$ & $11.7_{-2.9}^{+7.0}$ & 1.03 \\
$16: 30$ & $1.00 \pm 0.38_{-0.09}^{+0.08}$ & $1.00_{-0.08}^{+0.39}$ & 1.22 & $1.5 \pm 1.1_{-0.1}^{+0.3}$ & $1.8_{-0.5}^{+1.2}$ & 0.89 \\
$\eta^{\text {jet } 1}$ & $d \sigma_{b} / d \eta^{\text {jet } 1}$ & $d \sigma_{b}^{\mathrm{NLO}} / d \eta^{\text {jet } 1}$ & $C_{b}^{\text {had }}$ & $d \sigma_{c} / d \eta^{\text {jet } 1}$ & $d \sigma_{c}^{\mathrm{NLO}} / d \eta^{\text {jet } 1}$ & $C_{c}^{\text {had }}$ \\
\hline$-1.0:-0.25$ & $24.9 \pm 5.2_{-2.2}^{+1.9}$ & $17.5_{-2.7}^{+6.1}$ & 0.82 & $73 \pm 14_{-6}^{+13}$ & $99_{-26}^{+64}$ & 0.95 \\
$-0.25: 0.5$ & $47.6 \pm 8.2_{-4.2}^{+3.7}$ & $42.6_{-7.7}^{+12.7}$ & 1.01 & $177 \pm 24_{-15}^{+31}$ & $164_{-35}^{+75}$ & 1.05 \\
$0.5: 1.5$ & $49.3 \pm 7.8_{-4.4}^{+3.8}$ & $30.4_{-6.1}^{+7.9}$ & 0.91 & $71 \pm 17_{-6}^{+12}$ & $106_{-32}^{+41}$ & 1.04 \\
$1.5: 2.5$ & $23.7 \pm 5.5_{-2.1}^{+1.8}$ & $9.2_{-2.4}^{+3.6}$ & 0.76 & $8 \pm 15_{-1}^{+1}$ & $35_{-12}^{+23}$ & 1.01
\end{tabular}

Table 4: Differential cross sections for the most energetic jet as a function of $E_{T}^{\text {jet }}$ and $\eta^{\text {jet }}$ for the complete data set. For further details see the caption of Table 1.

\begin{tabular}{r|rcc|ccc}
$\begin{array}{c}E_{T}^{\mathrm{e} j e t} \\
(\mathrm{GeV})\end{array}$ & \multicolumn{1}{c}{$\begin{array}{c}d \sigma_{b} / E_{T}^{\mathrm{e} j e t} \\
(\mathrm{pb} / \mathrm{GeV})\end{array}$} & $\begin{array}{c}d \sigma_{b}^{\mathrm{NLO}} / E_{T}^{\mathrm{e} j e t} \\
(\mathrm{pb} / \mathrm{GeV})\end{array}$ & $C_{b}^{\text {had }}$ & $\begin{array}{c}d \sigma_{c} / E_{T}^{\mathrm{e} j e t} \\
(\mathrm{pb} / \mathrm{GeV})\end{array}$ & $\begin{array}{c}d \sigma_{c}^{\mathrm{NLO}} / E_{T}^{\mathrm{e} j e t} \\
(\mathrm{pb} / \mathrm{GeV})\end{array}$ & $C_{c}^{\mathrm{had}}$ \\
\hline $6: 10$ & $16.1 \pm 1.8_{-1.4}^{+1.2}$ & $12.3_{-3.0}^{+5.1}$ & 0.67 & $42.2 \pm 5.2_{-3.7}^{+7.3}$ & $64_{-18}^{+38}$ & 1.00 \\
$10: 15$ & $6.6 \pm 1.3_{-0.6}^{+0.5}$ & $5.4_{-1.1}^{+1.8}$ & 1.00 & $22.3 \pm 4.2_{-2.0}^{+3.9}$ & $19.6_{-5.5}^{+7.5}$ & 1.06 \\
$15: 30$ & $2.1 \pm 0.6_{-0.2}^{+0.2}$ & $1.08_{-0.26}^{+0.40}$ & 1.21 & $0.3 \pm 1.9_{-0.1}^{+0.1}$ & $1.7_{-0.5}^{+1.2}$ & 0.87
\end{tabular}

Table 5: Differential cross sections of $E_{T}^{e j e t}$ for the jet associated to the electron from beauty or charm decays for the complete data set. For further details see the caption of Table 1. 


\section{ZEUS}

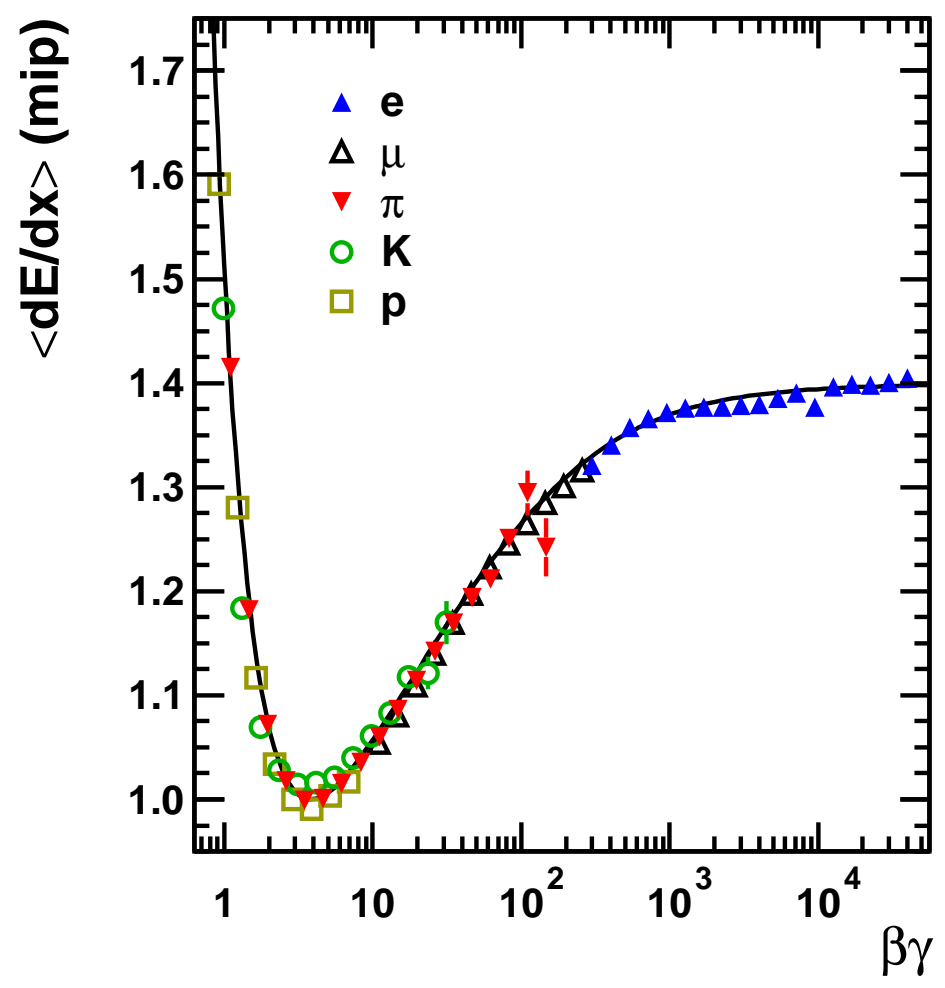

Figure 1: The mean $\mathrm{d} E / \mathrm{d} x$ measured in the CTD, $\langle\mathrm{d} E / \mathrm{d} x\rangle$, as a function of $\beta \gamma$ for different samples of identified particles as denoted in the figure. The curve shows a physically motivated parametrisation of the $\langle\mathrm{d} E / \mathrm{d} x\rangle$ dependence on $\beta \gamma$. 


\section{ZEUS}

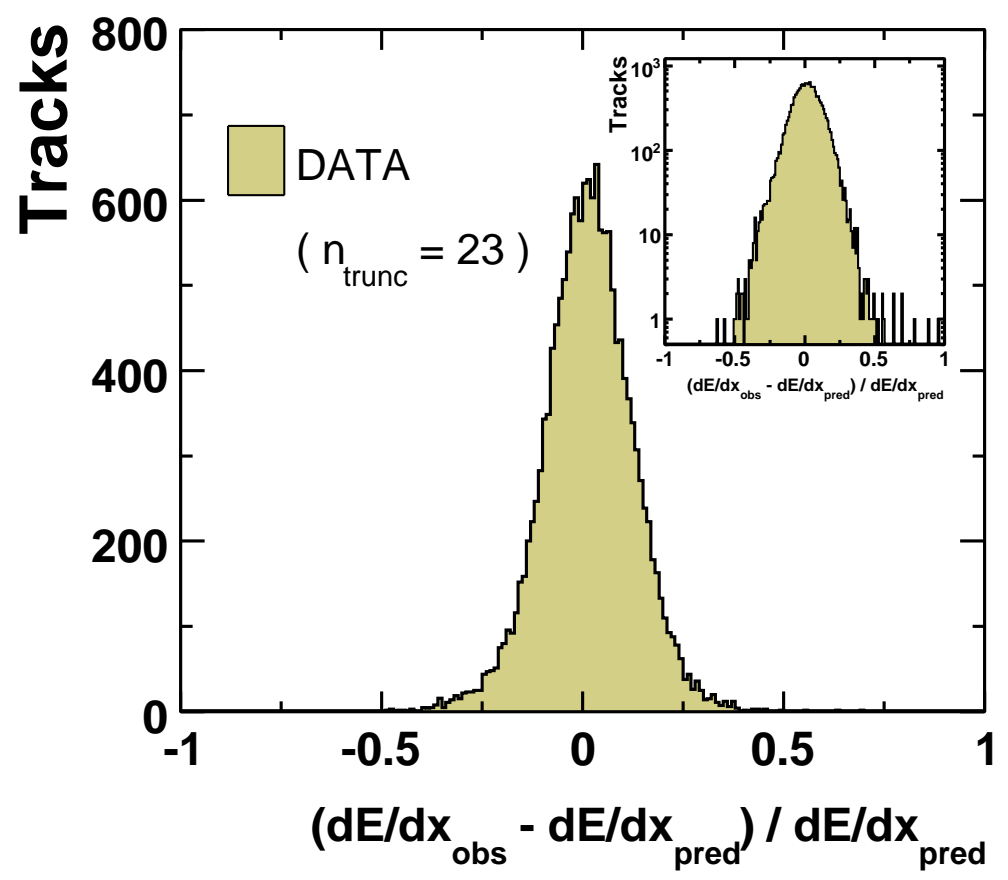

Figure 2: Distribution of the relative difference between the observed ( $\left.\mathrm{d} E / \mathrm{d} x_{\mathrm{obs}}\right)$ and predicted ( $\left.\mathrm{d} E / \mathrm{d} x_{\text {pred }}\right)$ specific energy loss for the track sample with $n_{\text {trunc }}=23$. The inset shows the same distribution with a logarithmic ordinate scale. 

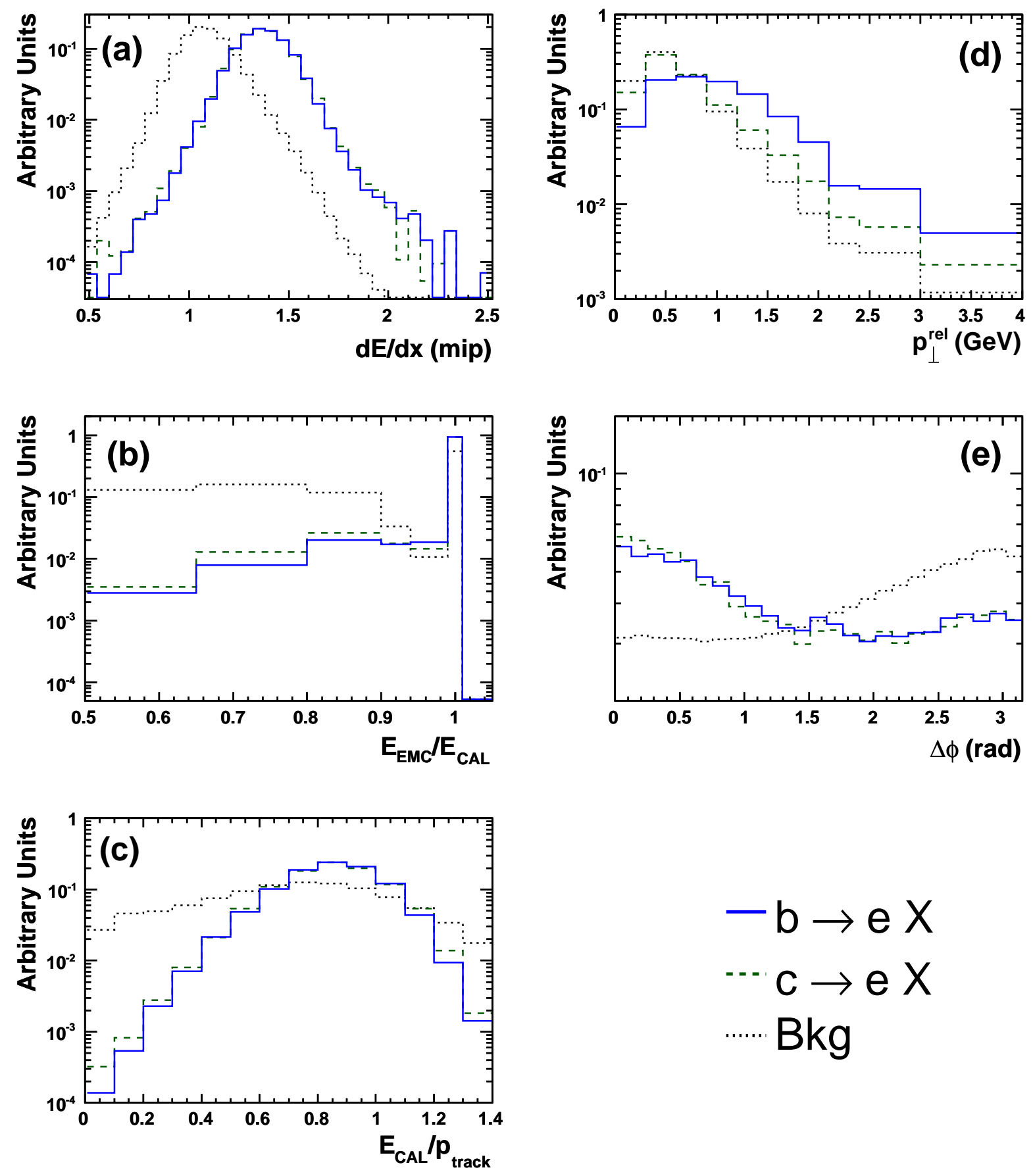

Figure 3: Normalised distributions of the five input variables used in the likelihood for the electron candidates extracted from the Monte Carlo, without applying the cuts on $\mathrm{d} E / \mathrm{d} x$ and $E_{\mathrm{EMC}} / E_{\mathrm{CAL}}$. The solid line shows the distribution for electrons from semileptonic b-quark decays, the dashed line for c-quark decays and the dotted line the background (Bkg). 


\section{ZEUS}

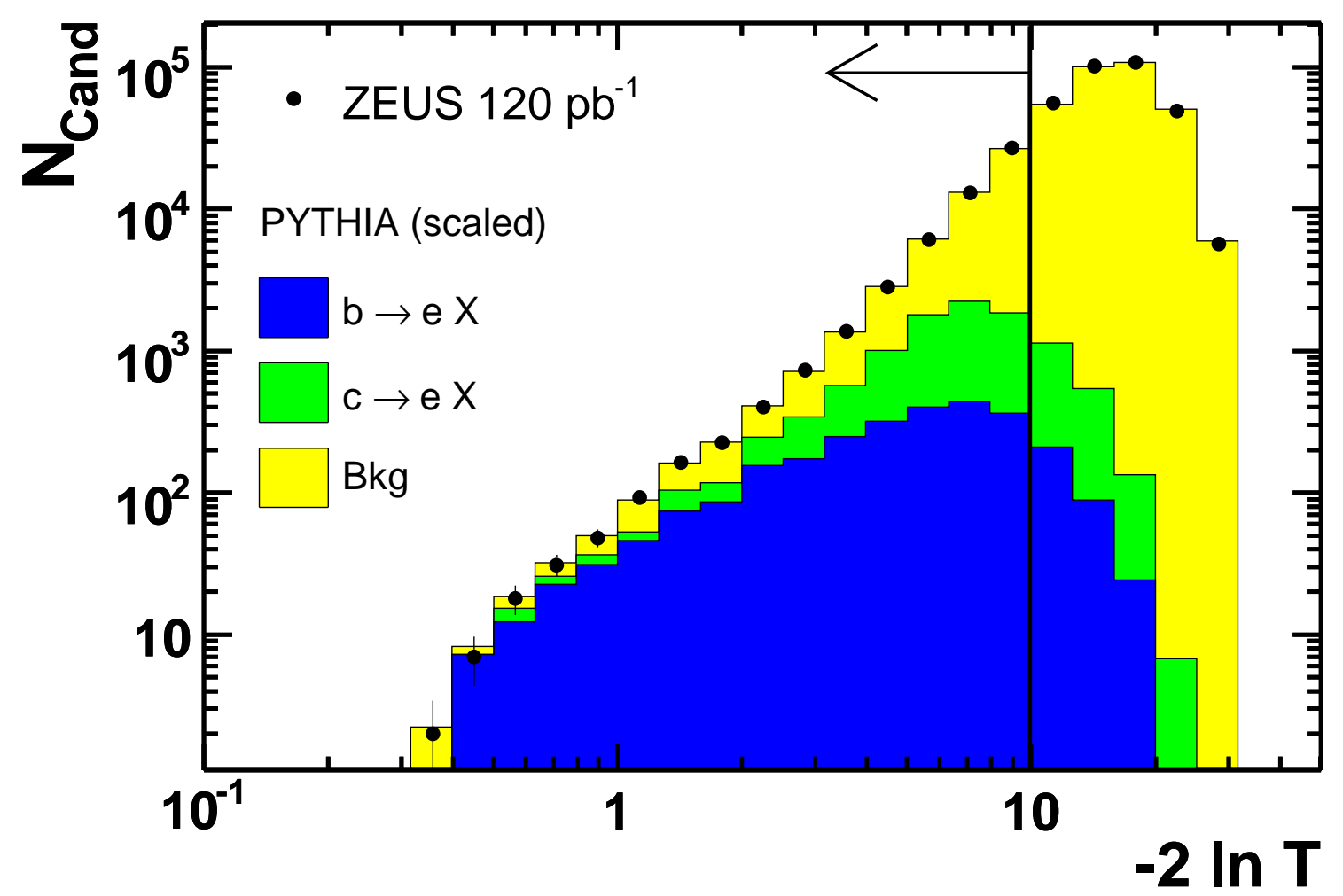

Figure 4: The distribution of the likelihood ratio for electron candidates, $N_{\text {cand }}$, in data compared to the Monte Carlo expectation after the fit described in the text. The arrow indicates the region included in the fit $(-2 \ln T<10)$. The shaded areas show the fitted contributions from $b$ quarks, $c$ quarks and background as denoted in the figure. 


\section{ZEUS}
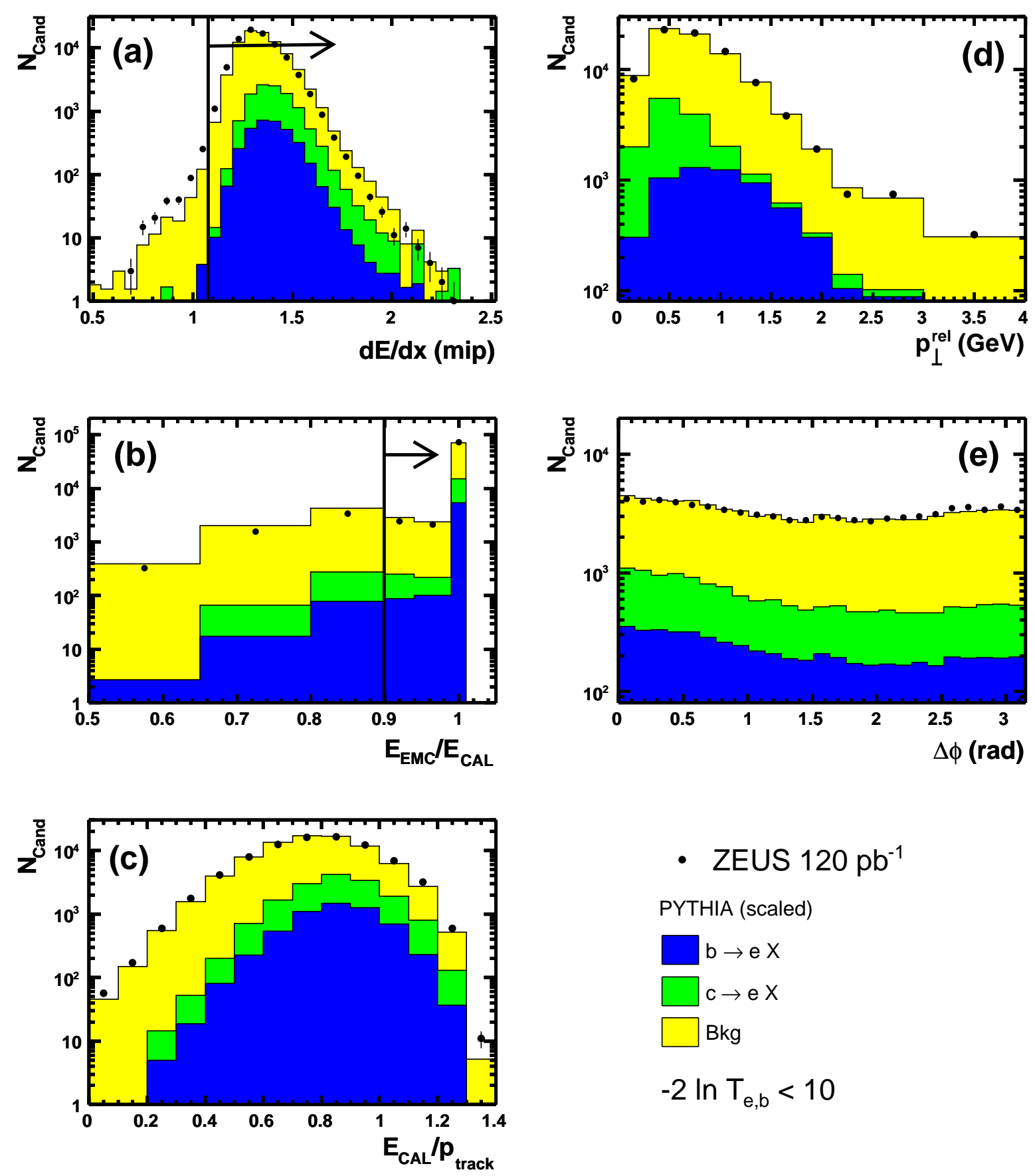

- ZEUS $120 \mathrm{pb}^{-1}$

PYTHIA (scaled)

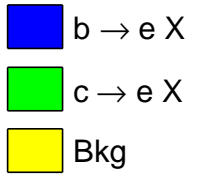

$-2 \ln T_{e, b}<10$

Figure 5: Distributions of the five input variables of the likelihood for the electron candidates used in the fit $(-2 \ln T<10)$. All cuts have been applied except $\mathrm{d} E / \mathrm{d} x>$ 1.1 in a) and $E_{\mathrm{EMC}} / E_{\mathrm{CAL}}>0.9$ in b) (the cuts are indicated in the figure). The shaded areas show the contributions from $b$ quarks, $c$ quarks and background as denoted in the figure, after applying the scale factors from the fit. 


\section{ZEUS}
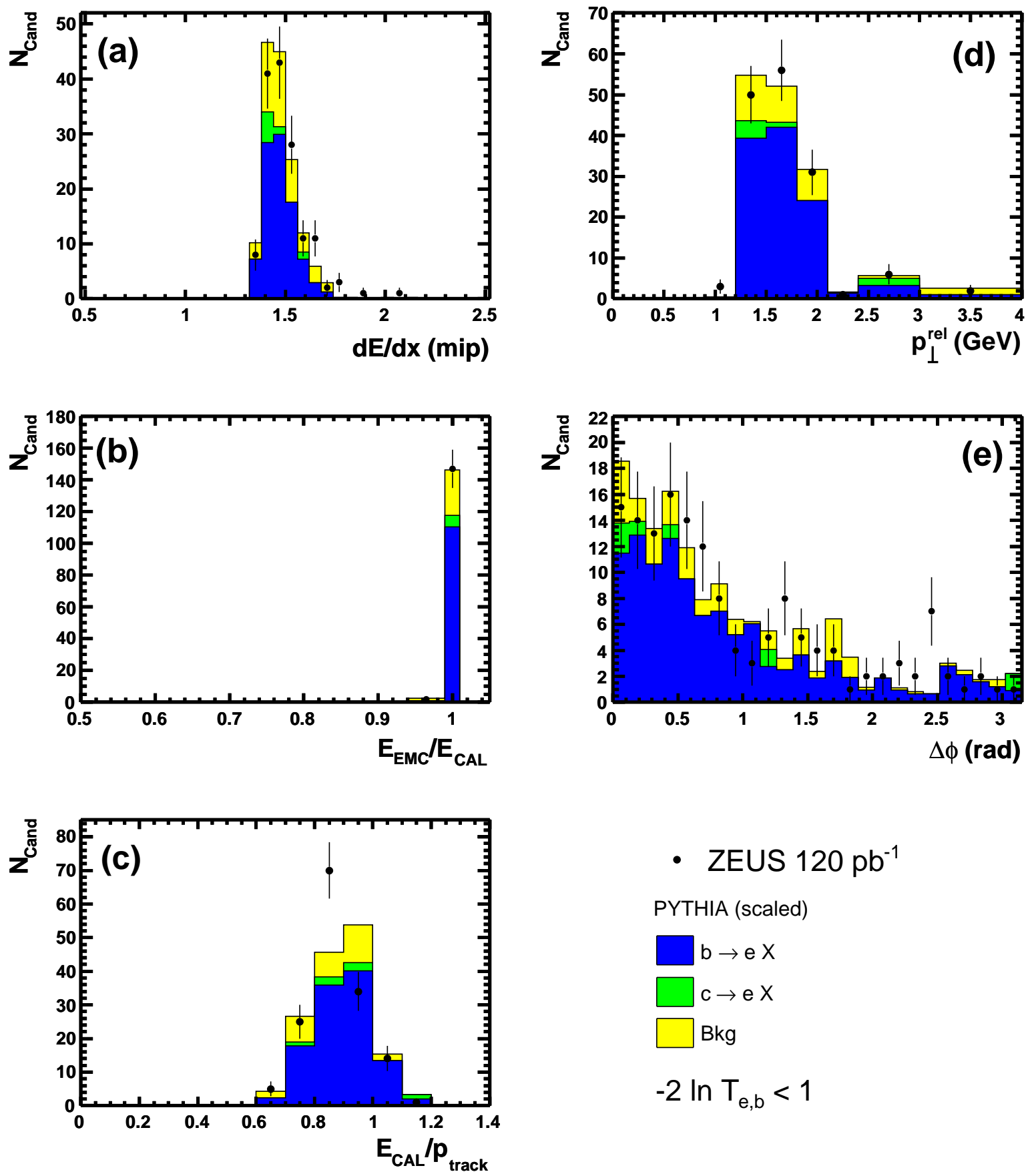

- ZEUS $120 \mathrm{pb}^{-1}$

PYTHIA (scaled)

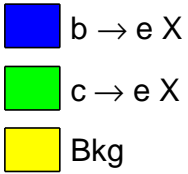

$-2 \ln T_{e, b}<1$

Figure 6: Distributions of the five input variables of the likelihood for a beauty enriched sample (candidates with $-2 \ln T<1$ ). In these plots the ordinate is shown on a linear scale. Other details as in the caption of Fig. 5. 


\section{ZEUS}
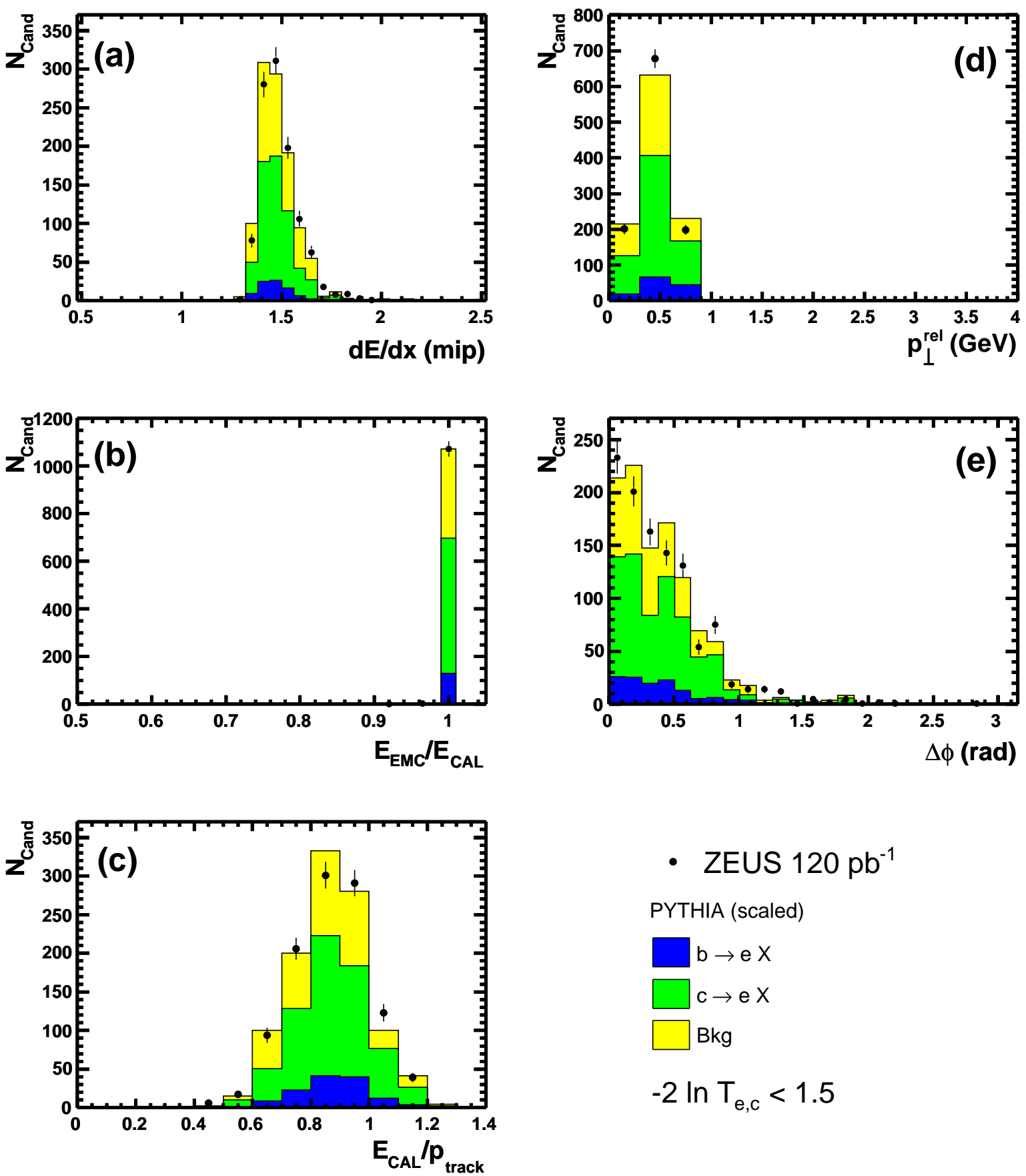

- ZEUS $120 \mathrm{pb}^{-1}$

PYTHIA (scaled)

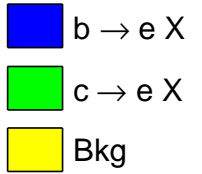

$-2 \ln \mathrm{T}_{\mathrm{e}, \mathrm{c}}<1.5$

Figure 7: Distributions of the five input variables of the likelihood for a charm enriched sample (candidates with $-2 \ln T_{e, c}<1.5$ ). In these plots the ordinate is shown on a linear scale. Other details as in the caption of Fig. 5. 


\section{ZEUS}
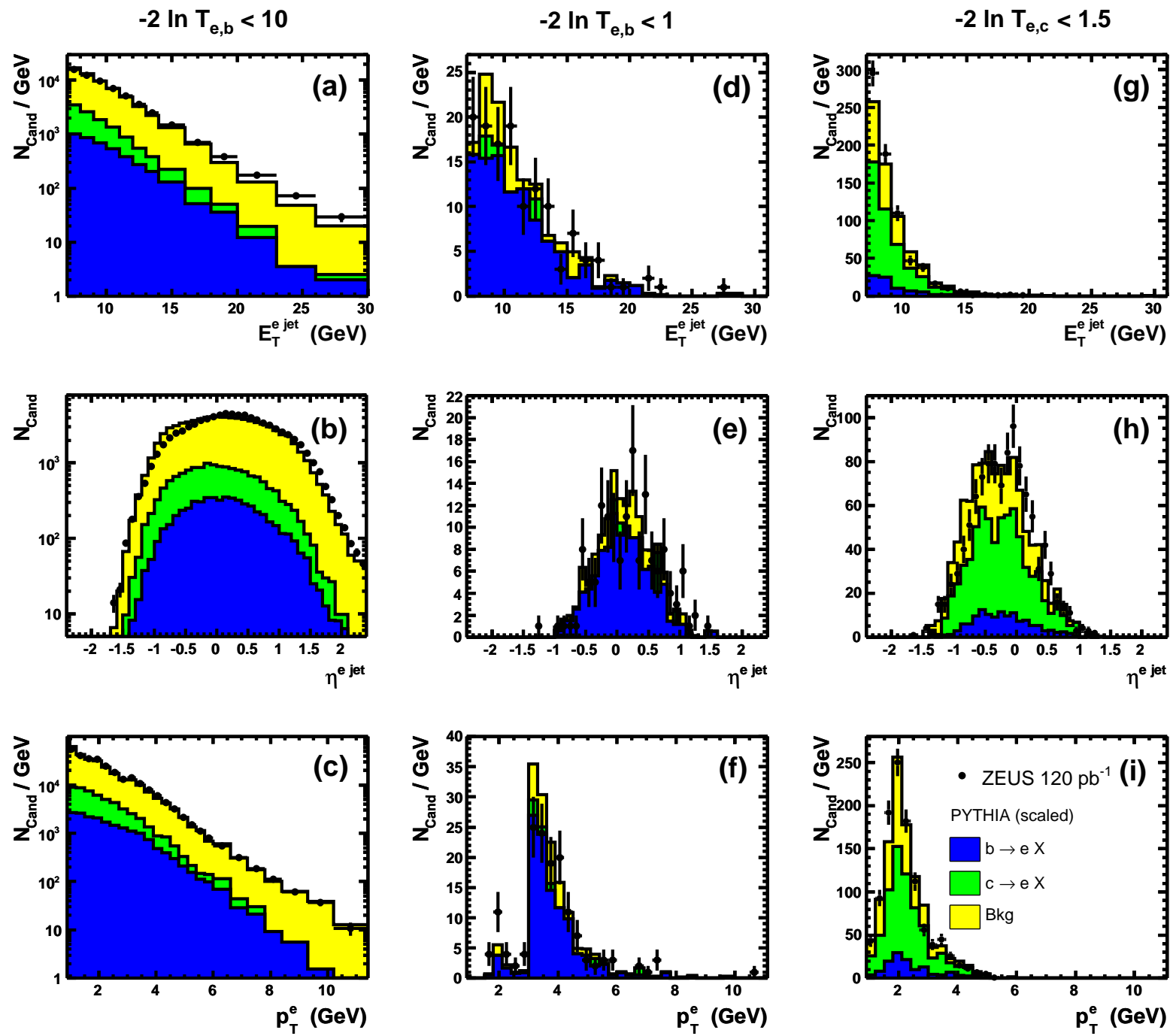

Figure 8: Distributions of $E_{T}^{e j e t}$ and $\eta_{T}^{e \text { jet }}$ of the jet associated with the electron, and $p_{T}^{e}$ of the electron candidate. Figures a)-c) contain all electron candidates satisfying $\left.-2 \ln T_{e, b}<10 ; d\right)-f$ ) and $g$ )-i) show the same distributions for the beauty $\left(-2 \ln T_{e, b}<1\right)$ and charm $\left(-2 \ln T_{e, c}<1.5\right)$ enriched samples, respectively. Other details as in the caption of Fig. 5. 


\section{ZEUS}

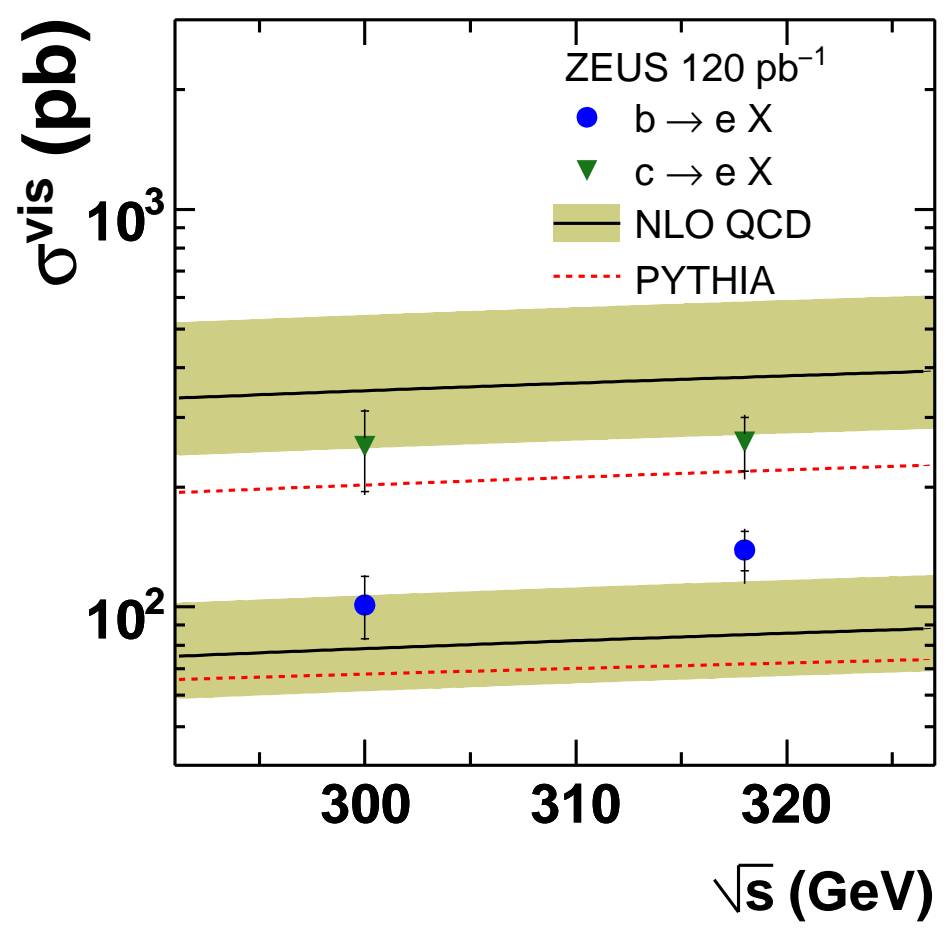

Figure 9: Total cross sections for electrons from $b$ and $c$ quarks in photoproduction events, $Q^{2}<1 \mathrm{GeV}^{2}$ and $0.2<y<0.8$, with at least two jets with $E_{T}>7(6) \mathrm{GeV}$, $|\eta|<2.5$ and the subsequent semileptonic decay to an electron with $p_{T}>0.9 \mathrm{GeV}$ and $|\eta|<1.5$. The measurements are shown as points. The inner error bar shows the statistical uncertainty and the outer error bar shows the statistical and systematic uncertainties added in quadrature. The solid line shows the NLO QCD prediction after hadronisation corrections, with the theoretical uncertainties indicated by the band; the dashed line shows the prediction from PYTHIA. 


\section{ZEUS}
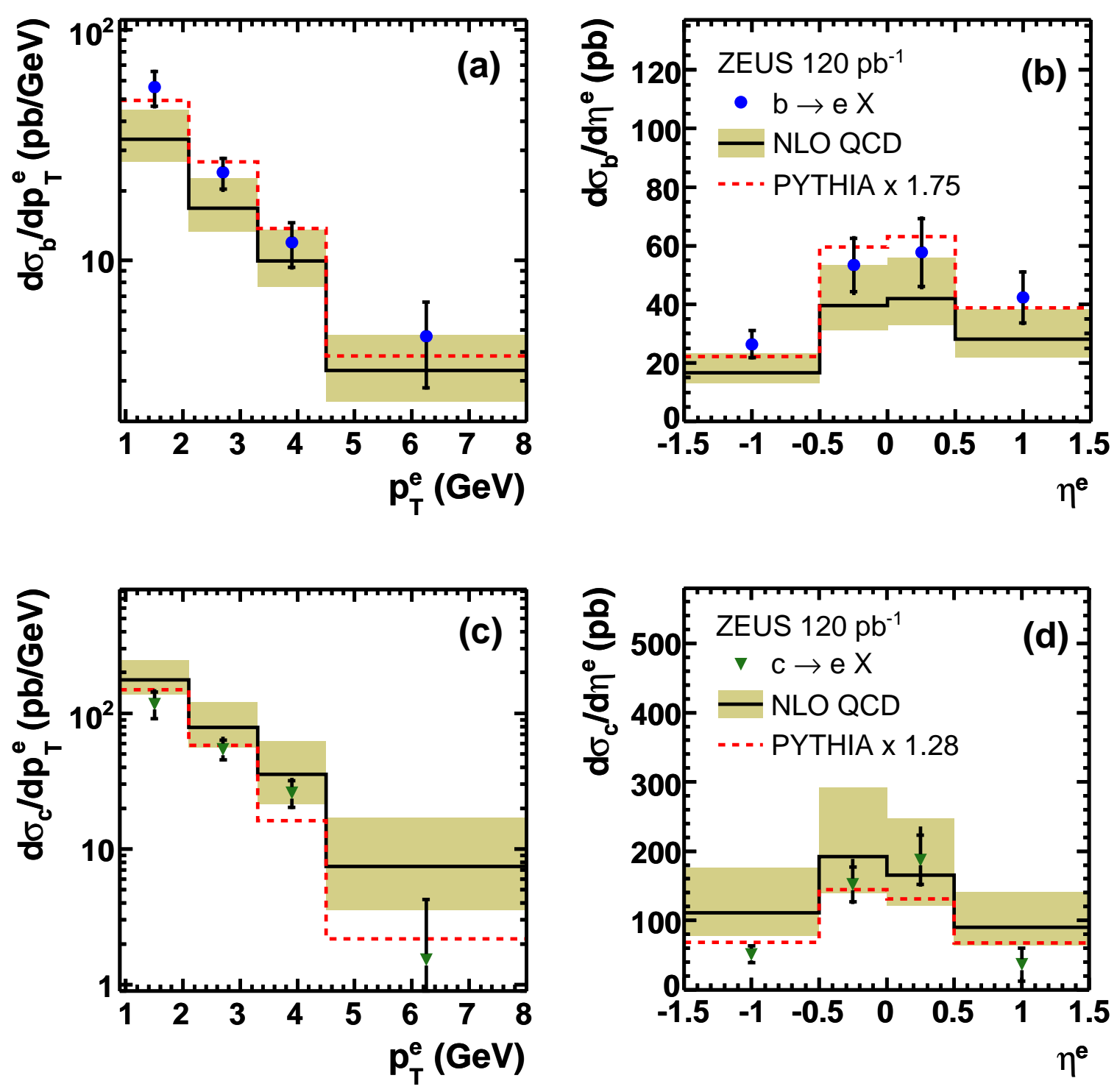

Figure 10: Differential cross sections as a function of a), c) the transverse momentum and $b)$, d) the pseudorapidity of the electrons. Plots a) and b) are for $b$ quark production while c) and d) are for c-quark production. The measurements are shown as points. The inner error bar shows the statistical uncertainty and the outer error bar shows the statistical and systematic uncertainties added in quadrature. The solid line shows the NLO QCD prediction after hadronisation corrections, with the theoretical uncertainties indicated by the band; the dashed line shows the scaled prediction from PYTHIA. 


\section{ZEUS}
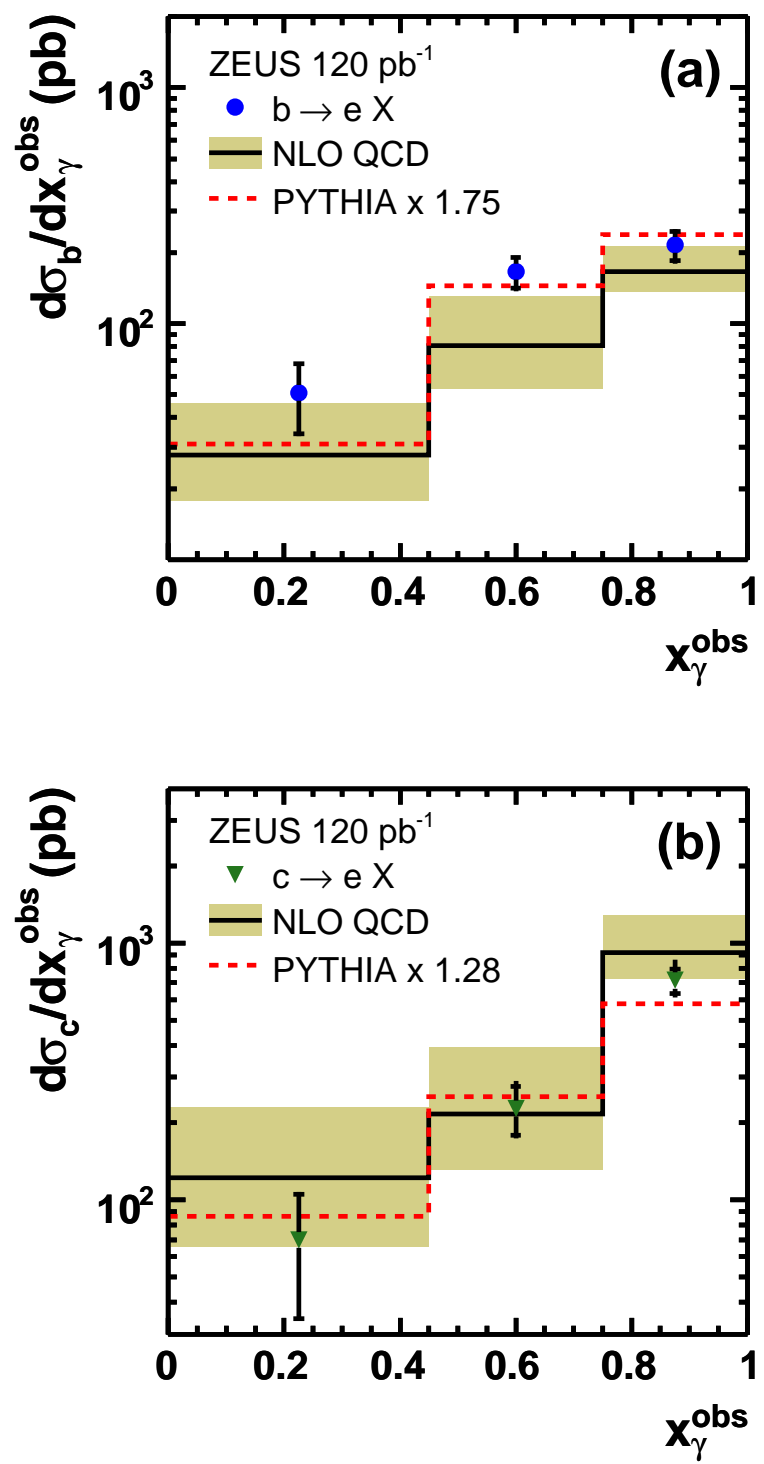

Figure 11: Differential cross sections as a function of $x_{\gamma}^{\text {obs }}$. a) shows the distribution for electrons from b-quark production while b) shows c-quark production. Other details as in the caption of Fig. 10. 


\section{ZEUS}
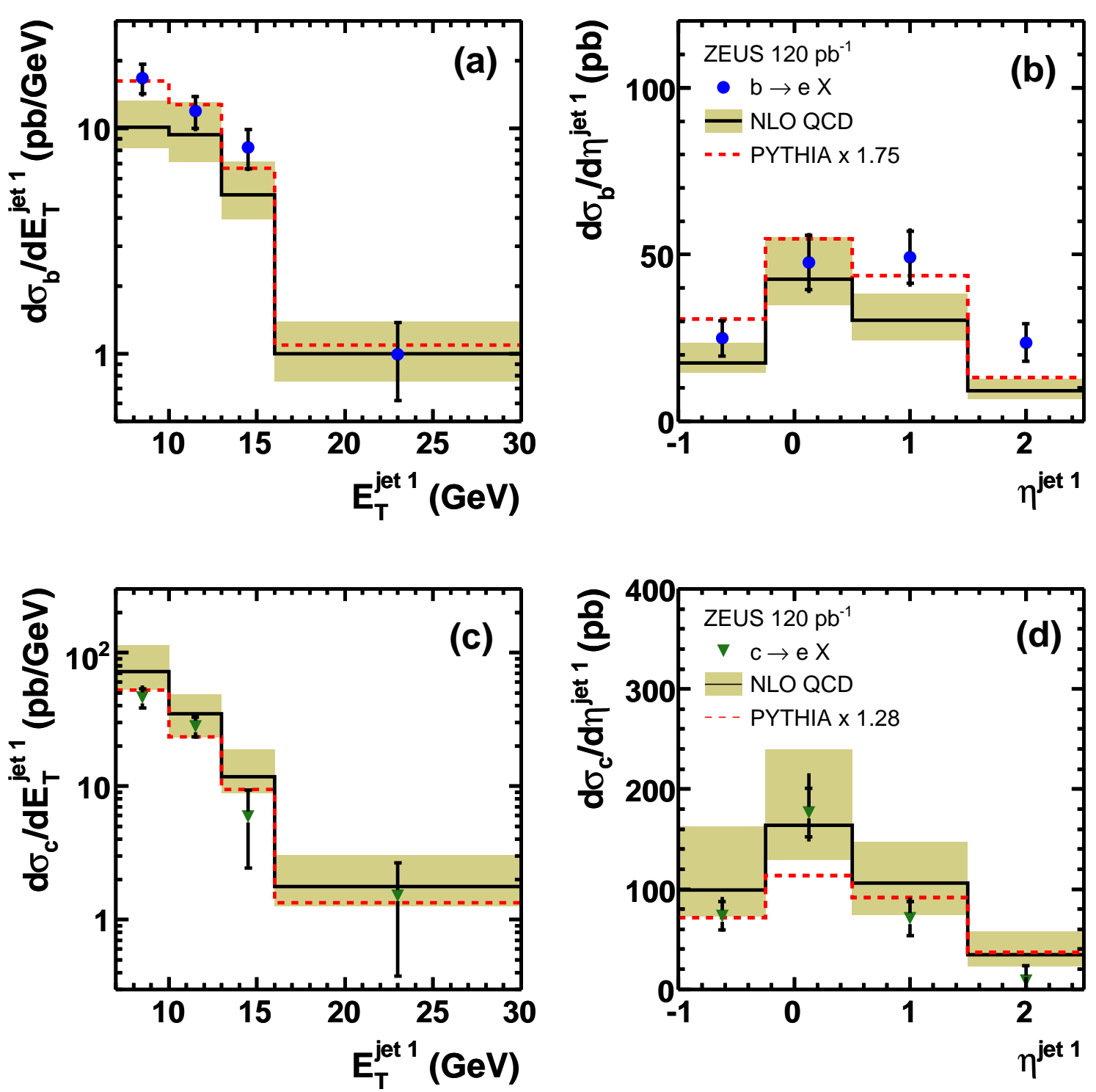

Figure 12: Differential cross sections as a function of a), c) the transverse energy and b), d) the pseudorapidity of the highest-energy jet. Plots a) \&5 b) show the distributions for electrons from b-quark production while plots c) \&f d) show those for c-quark production. Other details as in the caption of Fig. 10. 


\section{ZEUS}
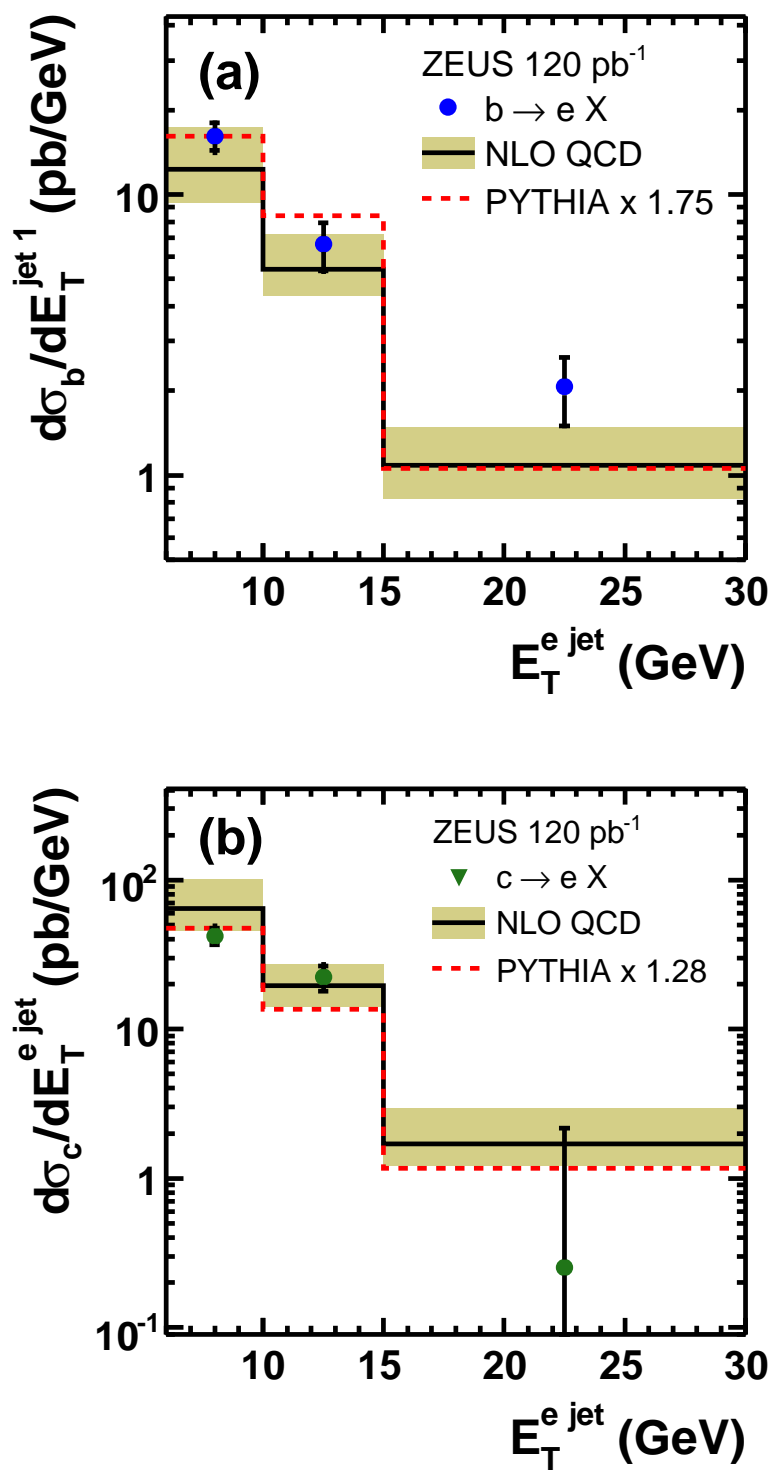

Figure 13: Differential cross sections for a) b-quark and b) c-quark production as a function of the transverse energy of the jet associated to the electron. Other details as in the caption of Fig. 10. 


\section{ZEUS}

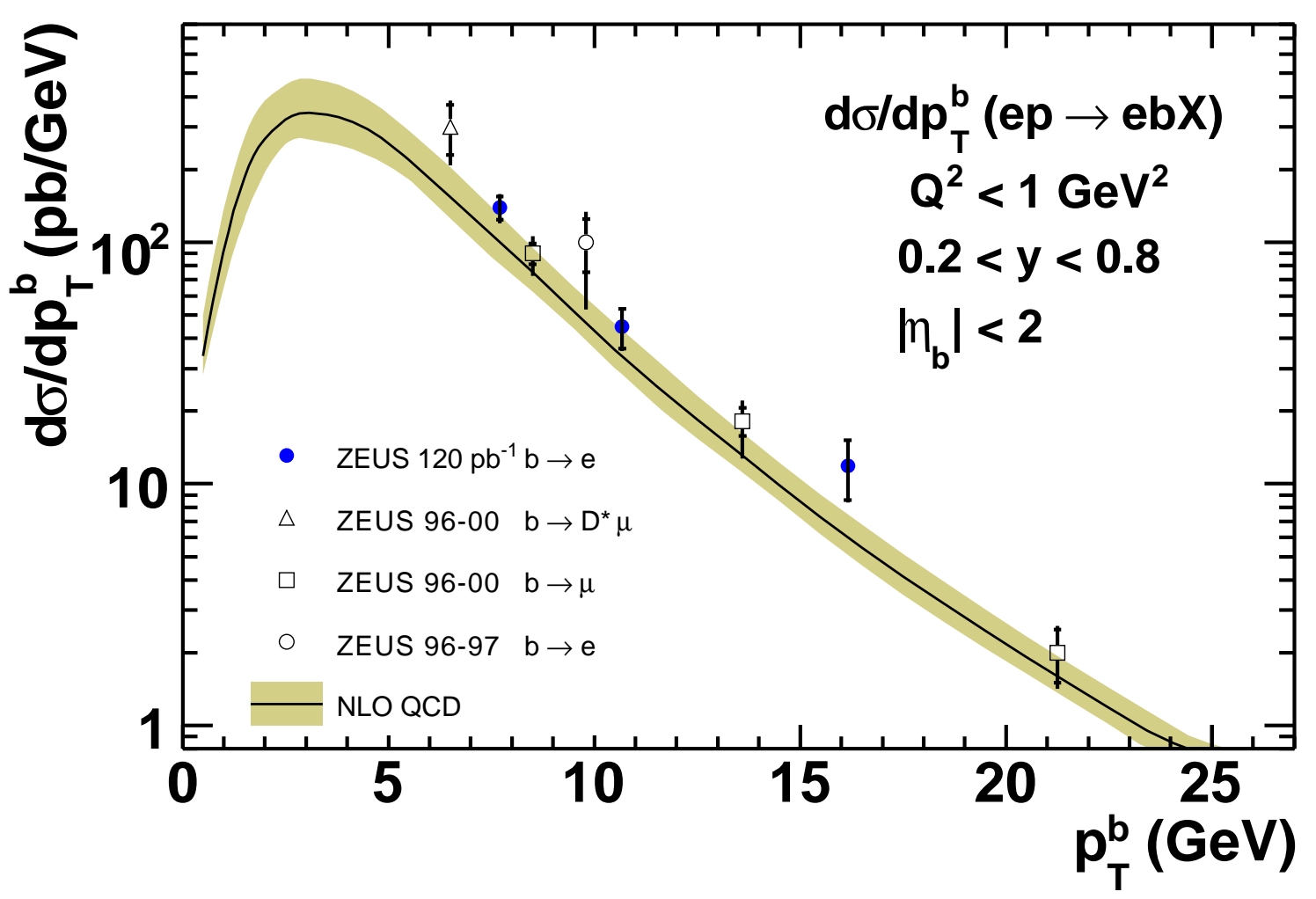

Figure 14: Differential cross section for b-quark production as a function of transverse momentum, $p_{T}^{b}$, compared to the results of previous ZEUS measurements as indicated in the figure. The measurements are shown as points. The inner error bar shows the statistical uncertainty and the outer error bar shows the statistical and systematic uncertainties added in quadrature. The solid line shows the NLO QCD prediction from the FMNR program with the theoretical uncertainty shown as the shaded band. 